\title{
Thymol polymeric nanoparticle synthesis and its effects on the toxicity of high glucose on OEC cells: involvement of growth factors and integrin-linked kinase
}

This article was published in the following Dove Press journal:

Drug Design, Development and Therapy

\author{
Elahe Karimi ${ }^{1,2}$ \\ Shahryar Abbasi ${ }^{1,3}$ \\ Naser Abbasi ${ }^{2,4}$ \\ 'Department of Chemistry, llam Branch, \\ Islamic Azad University, Ilam, Iran; \\ ${ }^{2}$ Biotechnology and Medicinal Plants \\ Research Center, llam University of \\ Medical Sciences, llam, Iran; ${ }^{3}$ Department \\ of Chemistry, Faculty of Science, Ilam \\ University, Ilam, Iran; ${ }^{4}$ Department of \\ Pharmacology, Medical School, Ilam \\ University of Medical Sciences, Ilam, Iran
}

Correspondence: Shahryar Abbasi Department of Chemistry, Faculty of Science, Ilam University, new campus of Ilam University, building 3, appt.304., Ilam, Iran

Tel/Fax +98 84I 2227022

Email abosina2000@yahoo.com

\begin{abstract}
Background: Nowadays, the drug delivery system is important in the treatment of diseases. Purpose: A polymeric nanoparticle modified by oleic acid (NPMO) as a Thymol (Thy) drug release system was synthesized from Thymbra spicata and its neurotrophic and angiogenic effects on rat's olfactory ensheathing cells (OECs) in normal (NG) and high glucose (HG) conditions were studied.

Methods: The NPMO was characterized by using different spectroscopy methods, such as infrared, HNMR, CNMR, gel permeation chromatography, dynamic light scattering, and atomic force microscopy. Load and releasing were investigated by HPLC. The toxicity against OECs diet-induced by MTT assay. ROS and generation of nitric oxide (NO) were evaluated using dichloro-dihydro-fluorescein and Griess method, respectively. The expression of protein integrin-linked kinase (ILK), vascular endothelial growth factor (VEGF), brain-derived neurotrophic factor (BDNF), and nerve growth factor (NGF) were evaluated by Western blotting.
\end{abstract}

Results: ThyNPMO is desirable for transferring drug as a carrier. The amount of Thy and extract (E) loaded on NPMO estimated at $43 \pm 2.5 \%$ and $41 \pm 1.8 \%$, respectively. Then, $65 \%$ and $63 \%$ of the drug load were released, respectively. Thy, ThyNPMO, E, and ENPMO prevented HG-induced OECs cell death (EC50 33 $\pm 1.5,22 \pm 0.9,35 \pm 1.8$, and $25 \pm 1.1 \mu \mathrm{M}$, respectively). Incubation with Thy, ThyNPMO, E ,and ENPMO at high concentrations increased cell death with LC50 105 $\pm 3.5,82 \pm 2.8,109 \pm 4.3$, and $86 \pm 3.4 \mu \mathrm{M}$, respectively in HG states.

Conclusion: OECs were protected by ThyNPMO and ENPMO in protective concentrations by reducing the amount of ROS and NO, maintaining ILK, reducing VEGF, and increasing BDNF and NGF. The mentioned mechanisms were totally reversed at high concentrations.

Keywords: thymol, polymeric nanoparticle, olfactory enshealing cell, drug delivery, high glucose

\section{Introduction}

Nanoscale drug carriers are generally synthesized from natural and polymeric materials for slow and targeted release in sizes of 10-1000 nm. Therefore, because the structure of drug carriers has important benefits such as biocompatibility and biodegradability, it is widely used to develop pharmaceutical industries. Nanoparticles (NP), either encapsulated or absorbed, carry the drug more effectively to be used in the target tissue. ${ }^{1}$ Today, anticancer effects are one of the most commonly used NPs in the treatment of diseases. ${ }^{2,3}$ 
Diabetes is one of the factors that can cause micro- and macrovascular disorders that increase mortality, lower quality of life, and increase the cost of treatment in the community. ${ }^{4}$ Increasing the cost of this disease returns to the destructive effects of oxidative stress in retinal degeneration, the renal system, and peripheral nerves. ${ }^{5}$ In addition, nitric oxide (NO) production has been shown to be closely related to oxidative stress (ROS) in diabetic conditions. ${ }^{6}$ The association between olfactory dysfunction in diabetic conditions has been reported by several previous studies. While studied and tested on olfactory cells, it could be a predictor of diseases as Parkinson, ${ }^{7}$ multi-infarct dementia and Alzheimer, ${ }^{8}$ multiple sclerosis. ${ }^{9}$ On the other hand, it has been shown that in diabetic conditions, by studying olfactory cells, prediction of micro- and macrovascular changes, and central diabetic neuropathy is earlier. ${ }^{10,11}$

It has been shown that under high glucose (HG) conditions, integrin-linked kinase (ILK) can play an important role in the pathogenesis of diabetic retinopathy due to vascular endothelial growth factor (VEGF) and intercellular adhesion molecule 1 (ICAM-1) effects. ${ }^{12}$

It also has been documented that serum levels of nerve growth factor (NGF) and brain-derived neurotrophic factor (BDNF) maybe are effective in the creation and development of diabetic peripheral neuropathy. ${ }^{13}$ Despite the protection of nerve cells as GLP-1 and DPP-IV inhibitors, which acts by inhibiting vascular leakage, medications that are effective in vascular dysfunction continue to be present in diabetes. ${ }^{14-16}$

Researchers have suggested that one of the effective ways to overcome the nervous and vascular disorders caused by diabetes is to use drug delivery techniques. ${ }^{17}$ One of the most inexpensive and biocompatible materials that is also used in the pharmaceutical industries and food is citric acid (CA). ${ }^{18}$ To synthesize a biocompatible polymer, in addition to CA, glycerol can also be used. ${ }^{19}$ To investigate the physicochemical properties of polymeric NP produced, Fourier-transform infrared (FTIR), nuclear magnetic resonance (NMR), gel permeation chromatography (GPC), dynamic light scattering (DLS), and atomic force microscopy (AFM) are used. ${ }^{20}$

The use of medicinal plant components in NP technology is very important in the treatment of diabetes. Aromatic medicinal herbs, such as the Thymus species, are used in traditional medicine for the treatment of various diseases. ${ }^{21-23}$ Thymol, a monoterpene, is found in many medicinal plant oils, such as Thymus vulgaris, Thymbra spicata, Thymus ciliates, Trachyspermum ammi, Monarda fistulosa, and Nigella sativa seeds. ${ }^{24}$ The American Food and Drug Administration has reported Thymol as an additive to food due to its nontoxicity and safety. ${ }^{25}$ Previously, the antidiabetic effects of Thymol on high-fat diet-induced diabetic mice have been reported. ${ }^{26}$ Thymol is bonded to a variety of NPs for various diseases. ${ }^{27,28}$ In this study, in addition to synthesizing and producing a polymeric NP and binding it with the active component of Thymbra spicata, Thymol, the effects and mechanisms of this drug delivery system in olfactory ensheathing cells (OECs) under HG and normal glucose (NG) were studied.

\section{Materials and methods}

\section{Preparation of polymeric nanoparticle modified by oleic acid (NPMO)}

By using CA (Carlo Erba) as AB3 monomer and glycerol (G) (Carlo Erba) as A3 monomer at different CA/G molar feed ratios, nano branching polymers (NP) were synthesized through the melt polycondensation. The mixture was cooled at room temperature. Viscose compound was dissolved in tetrahydrofuran (THF) (Merck) and filtered to obtain clear solution. The resulting solution was then concentrated under reduced pressure and was precipitated several times in cyclohexane solution (Merck). Precipitated compound was dissolved in THF and then placed in a dialysis bag (Mn cutoff 2000, Sigma-Aldrich) immersed in THF for $4 \mathrm{hrs}$ and THF in the outside medium of dialysis bag was replaced with the fresh solvent. Finally, to obtain pure product as colorless and viscose compound, THF was evaporated under the reduced pressure. ${ }^{20}$ In the second step, NPMO was synthesized. First, 3 g NP was poured into a polymerization capsule and then oleic acid was added. The resulting mixture was then heated at $90^{\circ} \mathrm{C}, 100^{\circ} \mathrm{C}, 120^{\circ} \mathrm{C}, 140^{\circ} \mathrm{C}$, and $160^{\circ} \mathrm{C}$, respectively, and stirred at $1000 \mathrm{rpm}$ magnetic stirrer for 5 hrs under the vacuum condition. To remove residue oleic acid, the mixture was washed several times with n-hexane. Then, it was washed in $20 \mathrm{~mL}$ ethanol. The produced polymer and ethanol solvent were put in a dialysis bag at room temperature for $4 \mathrm{hrs}$.

\section{FTIR spectroscopy}

The IR spectra of the NPMO was performed with a Nicolet 320 spectrophotometer FTIR which was prepared by mixing the fine powder with $\mathrm{KBr}$ and pressing. The spectra were obtained at a resolution of $4 \mathrm{~cm}^{-1}$ in the range $4000-500 \mathrm{~cm}^{-1} .^{29}$ 


\section{Nuclear magnetic resonance (NMR)}

All NMR experiments were conducted on a Bruker DRX $400(400 \mathrm{MHz})$ apparatus in $\mathrm{D}_{2} \mathrm{O}$ as solvent. Identical spectra were obtained by dissolving samples in $\mathrm{D}_{2} \mathrm{O}$ and the spectra were recorded at $500 \mathrm{MHz}$ (in ${ }^{1} \mathrm{H}$ and ${ }^{13} \mathrm{C}$ NMR spectra for all temperatures and concentrations). The resulting data were processed and analyzed using ACDLABS/1D NMR software. ${ }^{30}$

\section{Gel permeation chromatography (GPC)}

Molecular weights and distribution of the obtained NPMO were determined by means of Knauer GPC equipped with Smartline Pump 1000 with a PL Aqua gel-OH mixed-H 8 $\mu \mathrm{m}$ column connected to a differential refractometer, with water as the mobile phase at $25^{\circ} \mathrm{C}^{31}$

\section{Dynamic light scattering (DLS)}

DLS data were collected on Malvern Instruments Ltd., UK. The hydrodynamic diameters of NPMO in water were measured three times ( 5 run to each measurement) at $90^{\circ} \mathrm{C}$ to the incident beam. The reported values are number distribution intensities. The measurements were performed using the samples prepared by dispersing NPMO in $1 \mathrm{mM} \mathrm{NaCl}$ at $25^{\circ} \mathrm{C}$ at a ratio of $0.01 \%, \mathrm{w} / \mathrm{v}$. The mean size was accounted as the average of six measurements. ${ }^{32}$

\section{Atomic force microscopy (AFM)}

Using a Nanoscope IIIa Multimode scanning probe microscope (Ara-research Inc. Iran) for AFM, the morphology of the NPMO was determined. A droplet of the NPMO suspension was drying (freeze dryer) (Christ, Germany) onto a clean mica surface prior to AFM imaging. In tapping mode, images were scanned using silicon cantilevers (NSC15/AIBS) delivered by Micro Mash (Tallinn, Estonia), with a frequency around $300-330 \mathrm{kHz}$. The size of the images was $5 \times 5 \mu \mathrm{m}$. The images were scanned on at least six different areas of the sample. ${ }^{33}$

\section{Plant extraction and identification of Thymol by HPLC}

Thyme spicata aerial parts were collected from around Ilam, Iran in May 2018 during the flowering season. The identity of this plant was authenticated by the voucher specimens (NO 596) deposited in the Department of Horticulture, Faculty of Agriculture, Ilam University. After drying, the specimens were powdered and $20 \mathrm{~g}$ was used for extraction. Initially, the powder extracted by a Soxhlet extraction method in a water-ethanol solvent. The solvents in the extract were removed by rotary (IKA HB 10, Germany) device. The yield of extraction was $6.94 \%$ and then the extract was lyophilized and kept stored at $-20^{\circ} \mathrm{C}$. The lyophilized samples were dissolved in methanol and filtered through a $0.22-\mu \mathrm{m}$ syringe filter. ${ }^{34}$

HPLC method was done according to the reported procedure. ${ }^{35}$ A reversed-phase HPLC (Smart line; Knauer, Germany) with an ultraviolet detector (Well chrome, K2600; Knauer) and a C18 column (Nucleosil H.P.; 25 $\mathrm{cm} \times 0.46 \mathrm{~cm}$ internal diameter, $100 \AA$ pore size, particle size $3 \mu \mathrm{m}$, Knauer) using gradient elution with a UV absorbance detection was developed and validated for the determination of Thymol. Column temperature, mobile phase $(0.1 \%$ formic acid in water [B] was maintained at the range from $5 \%$ to $70 \%$ and solvent methanol [A]), flow rate, injection volume, and detection wavelength were set at $25^{\circ} \mathrm{C}, 1 \mathrm{~mL} / \mathrm{min}, 1 \mu \mathrm{L}$, and $284 \mathrm{~nm}$, respectively. In a similar condition, Thymol standard solution (dissolved in methanol) was run. A quantity of $250 \mathrm{mg}$ of dried extract was dissolved in $10 \mathrm{~mL}$ HPLC-grade methanol, sonicated for $15 \mathrm{mins}$, filtered and further diluted to $5 \mathrm{mg} / \mathrm{mL}$. The peaks obtained from the Thymbra spicata extract were compared with Thymol standard. A stock solution of Thymol standard was prepared at $0.1 \mathrm{mg} / \mathrm{mL}$ in HPLCgrade methanol, filtered and further diluted in the same solvent to obtain $15.6,31.25,62.5,125,250$, and $500 \mu \mathrm{M}^{36}$

\section{Encapsulation of Thymol and extract by NPMO}

One-tenth gram $\left(1.67 \times 10^{-2} \mathrm{mM}\right)$ of NPMO was dissolved in $5 \mathrm{~mL}$ distilled water and stirred for $1 \mathrm{hr}$. Then, Thymol (Sigma Aldrich) and extract dissolved in dimethyl sulfoxide (DMSO)(Merck) as stock solutions $(0.1 \mathrm{mM})$ were added dropwise to a NPMO mixture and various concentrations of Thymol and Thymol in extract were obtained $(25,50,100,150 \mu \mathrm{M})$. These solutions were sonicated at room temperature for obtaining the final product. ${ }^{37}$

\section{Load and releasing capacity}

HPLC method was used to determine the loading capacity, according to previous studies. ${ }^{38}$ To estimate the amount of pure Thymol and Thymol in extract loaded on NPMO, the HPLC was used. The mobile phase was made up of $40 \%$ methanol and $60 \%$ aqueous solution of formic acid (0.1\%). After $1 \mathrm{hr}$ sonication for encapsulation, water solutions of NPMO-pure Thymol and NPMO-Thymol in extract were prepared. To 
remove the non-encapsulated pure Thymol and Thymol in extract residue, the solution was centrifuged at 10,000 rpm and, after precipitation, the supernatant was filtered. ${ }^{39}$ An aliquot of the solution after filtration was injected into the HPLC to determine the concentration of encapsulation.

In vitro release of pure Thymol and Thymol in extract from NPMO was carried out by dissolving $5 \mathrm{mg}$ of pure Thymol and Thymol in extract loaded NPMO in $3 \mathrm{~mL}$ of PBS (0.1 M, pH 7.4). The NPMO solutions containing the pure Thymol and Thymol in extract $(1 \mathrm{~mL})$ were manipulated. At the time of sampling, the release medium was replaced by a fresh buffer subjected to HPLC for analysis. Each sample was then injected into the HPLC. ${ }^{40}$ Limit of detection (LOD) and limit of quantitation (LOQ) were 52.09 and 173.63 , respectively.

\section{Primary culturing OECs from the olfactory mucosa}

Olfactory mucosa lamina propria was used in 7-day-old rat's pups for isolation high proliferation cells. A mixture of ketamine/xylazine $(60 / 6 \mathrm{mg} / \mathrm{kg})$ was used for anesthesia. Then, the lower jaw and hard palate of the rat was removed and the nasal septum was separated with entry to the area of the nose. With fresh PBS containing 1\% of antibiotics, it was washed several times and then continued washing without antibiotics. About a third of the posterior septum that contains the olfactory mucosa consisting of olfactory epithelium and lamina propria was separated and then transferred to a sterile Petri dish. For isolation of cells, $0.25 \%$ trypsin enzyme (GIBCO) was used for 10 mins and then adding FBS (GIBCO). Then, the suspension was centrifuged for $10 \mathrm{mins}$ at $2000 \mathrm{rpm}$. The plate cells were suspended in $4 \mathrm{~mL}$ of DMEM/F-12 (GIBCO), containing FBS5\% and Penicillin-streptomycin solution 1\% (GIBCO) and forskolin (Sigma-Aldrich) mitogenic factor $5 \mu \mathrm{M}$ and after transfer to the flask, they were stored in an incubator at $37^{\circ} \mathrm{C}$ and carbon dioxide $5 \%$. The flasks were replaced $48 \mathrm{hrs}$ after cultivation to remove fibroblast cells. The cells begin to stick to the bottom of the flask with two different appearances: spindle-shaped Schwann-like cells and astrocyte-like cells with a flat appearance with multiple redundancies. After 10-12 days of culture, when the bottom of flask was completely filled with proliferated cells, fourth Cell Passage was performed. ${ }^{41}$ The cultured cells were exposed to $\mathrm{NG}$ (glucose $5.5 \mathrm{mM}$ ), HG (glucose $30 \mathrm{mM})$, or mannitol (30 mM) at 24, 48 and $72 \mathrm{hrs}$ and 48 hrs was selected.
The ethical approval for this study was obtained from the Animal Care and Ethics Committee (ACEC) of the Ilam University of Medical Science (IR.MEDILAM. REC.1396.84). According to ACEC recommendations, we tried our best to minimize research animal pain and suffering. To minimize the effects of transportation-induced physiological changes in subsequent biomedical research, it is advisable to consider two factors. According to the first factor, in the present study, it was noted that the animal transfer according to the physiological conditions in accordance with the international protocols with the least stress of the animal is carried out and according to the second factor, in general, mediators of stress response to reach the desired conditions (for example, for $24 \mathrm{hrs}$ ) were considered.

\section{MTT assay}

The cell viability and toxicity of the drug delivery system of various concentrations were evaluated by the MTT method. ${ }^{42}$ Based on this method, the OECs $(3 \times 104$ cells per well) were plated onto 96-well culture plates with different concentrations $(5,10,20,40,80,120$, and 180 $\mu \mathrm{m})$ of Thy, ThyNPMO,E, and ENPMO. The cultured cells were kept in incubator for 24,48 , and $72 \mathrm{hrs}$ in $5 \%$ carbon dioxide and humidity in $37^{\circ} \mathrm{C}$. Then, $10 \mu \mathrm{L}$ of the MTT labeling reagent was added and then the plates were incubated for $4 \mathrm{hrs}$. The final product, formazan, was dissolved in $100 \mu \mathrm{L}$ of DMSO and after being shaken at $37^{\circ} \mathrm{C}$; its absorption was measured at $594 \mathrm{~nm}$ with an ELISA reader (Spectra MAX; Molecular Devices, USA).

\section{Detection of ROS}

The amount of intracellular reactive oxygen species was measured by 2', 7'-dichlorofluorescein diacetate (DCFHDA) probe. ${ }^{43}$ OEC cells $\left(3 \times 10^{4}\right.$ cells/well $)$ incubated with $10 \mu \mathrm{M}$ DCFH-DA at $37^{\circ} \mathrm{C}$ for $30 \mathrm{mins}$ and in fluorescent plate reader (Biotek-FLx800) analyzed at $485 \mathrm{~nm}$ excitation and $520 \mathrm{~nm}$ emissions. An $\mathrm{H}_{2} \mathrm{O}_{2}$ standard (10-200 $\mathrm{nM}$ ) curve was used for ROS production.

\section{Assay of $\mathrm{NO}$}

By Griess reaction, the amount of generation of NO (nitrite) was assessed. ${ }^{44}$ After $48 \mathrm{hrs,} \mathrm{the} \mathrm{cells}\left(3 \times 10^{4}\right.$ cells/well) were treated with various concentrations $(20$, 40, 80, and 120) of Thy, ThyNPMO, E, and ENPMO. Then, $50 \mu \mathrm{L}$ of the culture supernatant was mixed with $50 \mu \mathrm{L}$ of the Griess reagent and incubated for 10 mins at room temperature. Absorbance calculated at $550 \mathrm{~nm}$ in a 
plate reader (Dynex MRX, USA); and sodium nitrate standard concentrations solutions were used as standard.

\section{Investigating the expression of ILK, VEGF, BDNF, and NGF proteins by Western blot}

The Western blot was explained according to what was previously tested. ${ }^{45}$ Treated OEC cells were homogenized under cold conditions. The culture cell suspension was prepared in lysis buffer. Homogeneous cell suspensions were centrifuged at $13,000 \times \mathrm{g}$ for $20 \mathrm{mins}$ at $4^{\circ} \mathrm{C}$, and until used, the supernatants were stored at $-80^{\circ} \mathrm{C}$. Content of total protein was measured according to the Micro BCA procedure (Pierce, Rockford, IL, USA). For electrophoresis, the equal amount of protein $(3000 \mu \mathrm{g})$ in each sample was loaded onto a polyacrylamide gel (10\%). The proteins were electrotransferred to the PVDF membranes and then blocked with 5\% skim milk and $0.1 \%$ Tween-20 in Tris-buffered saline at room temperature for $1 \mathrm{hr}$. The membranes were first incubated with the appropriate primary antibody and then conjugated to the secondary antibody. Using the ECL kit (Amersham Pharmacia Biotech Inc., Piscataway, NJ, USA), chemiluminescence detection of the immune complexes was performed, and the results were then quantified (Bio-Rad, USA). Protein analysis was performed with anti-human ILK (ab-76468, Priab 1/5000, Sec ab1/2000), VEGF, BDNF (SAB4300702, 1: 750, Sigma), and NGF (ab- P5498, Priab 1/500, Sigma).

\section{Statistical analysis}

Each test was repeated at least three times, with essentially identical results. Statistical analyses between different treatments or groups were determined using two-sample $t$-tests and ANOVA. Data were expressed as mean \pm SD. For all tests, $P$ values of $<0.05$ were considered significant. Terms half maximal effective concentration (EC 50) and half maximal lethal concentration (LC 50) refer to the concentration of a chemical, drug, or toxic substance that produces a response halfway between the baseline and maximum after a specified exposure time.

\section{Results}

\section{Evaluation of hyperbranched polyester}

Using spectroscopic and thermal analysis methods, including IR spectra, ${ }^{1} \mathrm{H}$, and ${ }^{13} \mathrm{C}$ NMR spectra, the structure of the synthesized NPMO was evaluated. The molecular weight was determined using GPC. By using DLS, the particle size of the NPMO in water was determined. Using AFM, the morphology of the NPMO was determined.

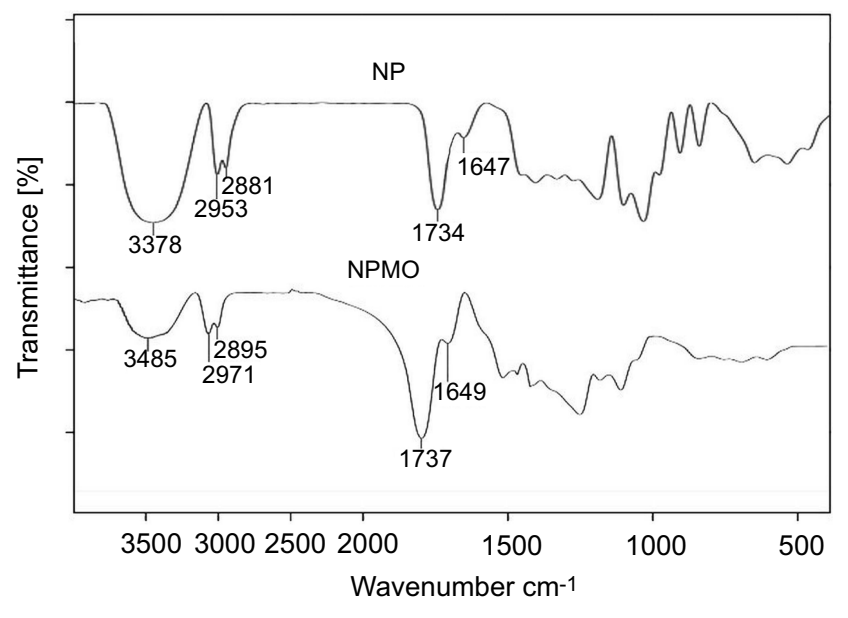

Figure I FTIR spectrum of polymeric nanoparticles (NP) and polymeric nanoparticles modified by oleic acid (NPMO).

The IR spectrum of the NP compound is shown in Figure 1. The peak appearing at $1734 \mathrm{~cm}^{-1}$ is related to the carbonyl ester group resulting from the combination of glycerol and CA. The $3375 \mathrm{~cm}^{-1}$ peak also indicates the abundance of hydroxyl groups in the NP compound. In the IR spectrum, the NPMO composition is also given. The peak of the carbonyl group appears at $1737 \mathrm{~cm}^{-1}$. This displacement in this spectrum is due to the formation of a new carbon derived from the carbonyl group, which is derived from the combination of hydroxyl groups of the NP compound with the oleic acid. As shown in the figure, after the addition of the oleic acid group (due to the presence of a long chain of carbon) to the NP compound, the peak strength of the hydroxyl group in the NPMO composition decreased to the NP compound, with a peak of $3485 \mathrm{~cm}^{-1}$.

The ${ }^{1} \mathrm{HNMR}$ spectrum of the NPMO compound is shown in Figure 2. For a more straightforward interpretation, these combinations of hydrogens with identical positions are named with the same letters. Hydrogen A is the hydrocarbon content of the monomeric CA present in the compound, a single peak at the highest intensity at $4.37 \mathrm{ppm}$. Hydrogen $\mathrm{B}$ is actually a single hydrogen-related monomer glycerol, which appears at 3.87 ppm. Hydrogen $\mathrm{C}$ is a methylated glycerol, which has two separate couriers due to the presence of neighbor hydrogen. Hydrogen D is related to the methane adjacent to the carbonyl group in the oleic acid monomer, split into a triplet peak at $2.45 \mathrm{ppm}$ due to the presence of two hydrogen neighbors. Hydrogen $\mathrm{E}$ is a methyl group adjacent to hydrogen $\mathrm{D}$, which has been split into a five-pixel peak due to the presence of four hydrogen neighbors. Hydrogen, named after the letter " $F$ ", appears in a single pixel at 2 ppm because of the same space-spatial position. Because of the dual-bonded proximity to hydrogen, I hydrogen has a different position with 


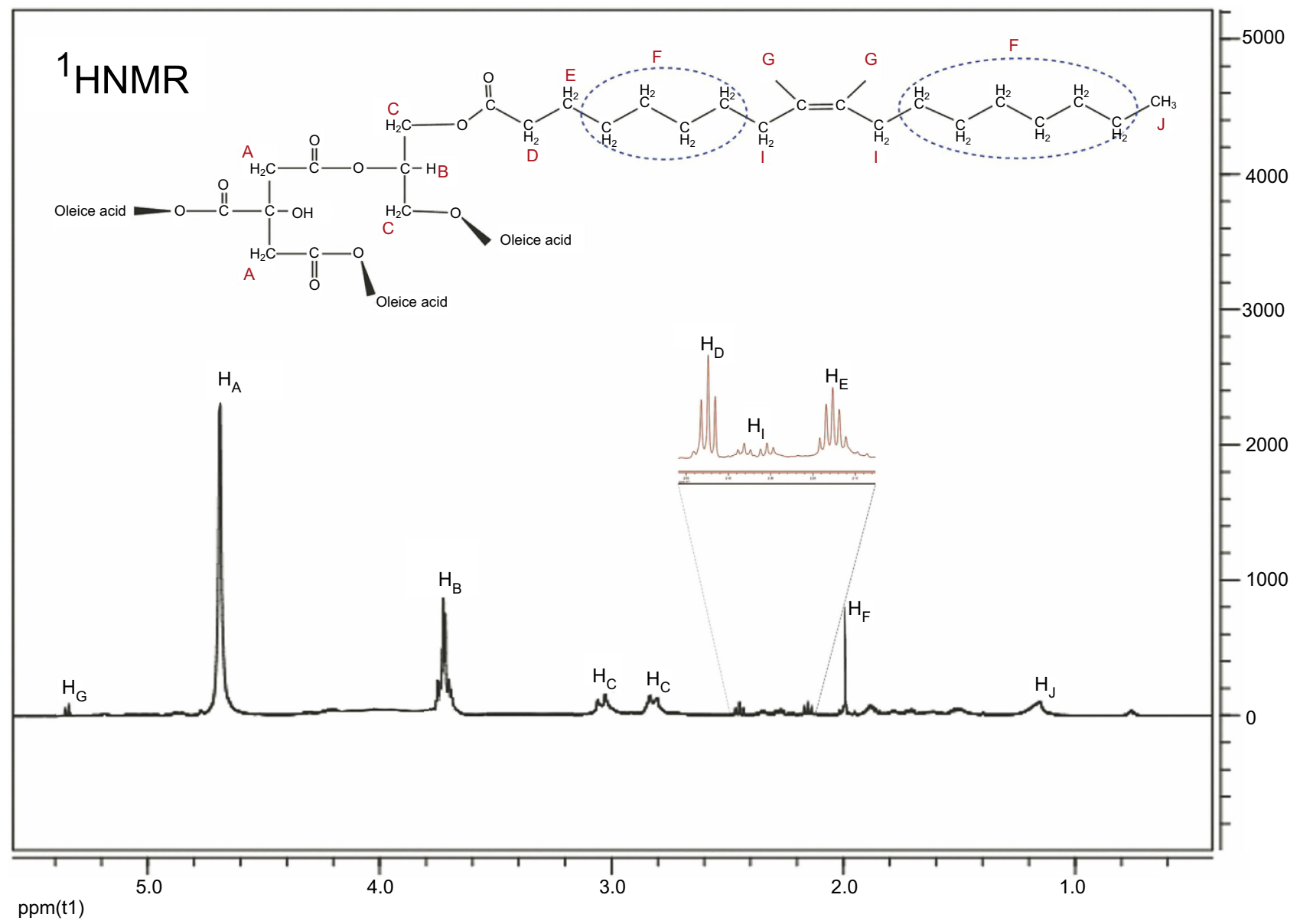

$\mathrm{ppm}(\mathrm{t} 1)$

Figure 2 'HNMR spectrum of polymeric nanoparticles modified by oleic acid (NPMO) in D2O.

neighboring hydrogen and appears as a peak in the range of 2.2-2.3 ppm. The G-hydrides appear to be in the form of a double peak at $5.26 \mathrm{ppm}$ due to the presence on the double bond, and eventually, the J-hydrides belong to the end-methyl group of the oleic acid chain, which, with minimal displacement, is a multiplicative peak at 1.15 ppm appear.

The ${ }^{13} \mathrm{CNMR}$ spectrum of the NPMO compound is presented in Figure 3. Regarding the fact that there are 12 types of carbon in the synthetic composition, in the CNMR spectrum, this combination has a 12-peak index in the carbon compounds of this composition, which, according to the form, is named all the carbons with the letters $\mathrm{A}-\mathrm{M}$, each of which are on the spectrum. Equivalent carbon is indicated by a letter as described below. Carbon $\mathrm{A}$ is the middle carbonic acid group of $\mathrm{CA}$, which appears at the end of the spectrum with a displacement of 177 ppm. Carbon B is the fourth type of CA that appears at $42 \mathrm{ppm}$. Methionine CA carbonated with the letter $\mathrm{C}$ appears at $28 \mathrm{ppm}$. The two Carboniferous carbon represented by the letter $\mathrm{D}$ appear at $172 \mathrm{ppm}$. The middle carbon of glycerol is marked with the letter $\mathrm{E}$, which is given at 73 ppm. Methyl glycerol carbonates have been identified with the letter $\mathrm{F}$, which appear as a peak at the highest intensity of $68 \mathrm{ppm}$. Carbon is the carbonyl oleic acid group with the letter $\mathrm{G}$, which has the largest displacement and peak at 183 ppm. Carbonyl adjacent carbonyl oleic acid group has a specific displacement due to its direct connection with the carbonyl group, with a different displacement than its other neighboring carbon, with the letter $\mathrm{H}$ marked with a peak of 38 ppm. Carbon labeled with the letter "I" has a roughly identical position and appears as a peak of more intensity than the other oleic acid carbon at $26 \mathrm{ppm}$. The adjacent dual-carbon carbons are positioned differently from other neighboring carbon and are marked with the letter $\mathrm{J}$ and have a peak at $21 \mathrm{ppm}$. The carbons that are connected by a double bond are named with the letter $\mathrm{K}$ and appear as a peak at $130 \mathrm{ppm}$. At the end of the carbon, the methyl group has appeared with a minimum displacement of $9 \mathrm{ppm}$.

The GPC diagram of NPMO is shown in Figure 4. This molecular weight chart is equivalent to $4221.72 \mathrm{~g} / \mathrm{mol}$ for synthetic composition. Also, the single-medal of the 


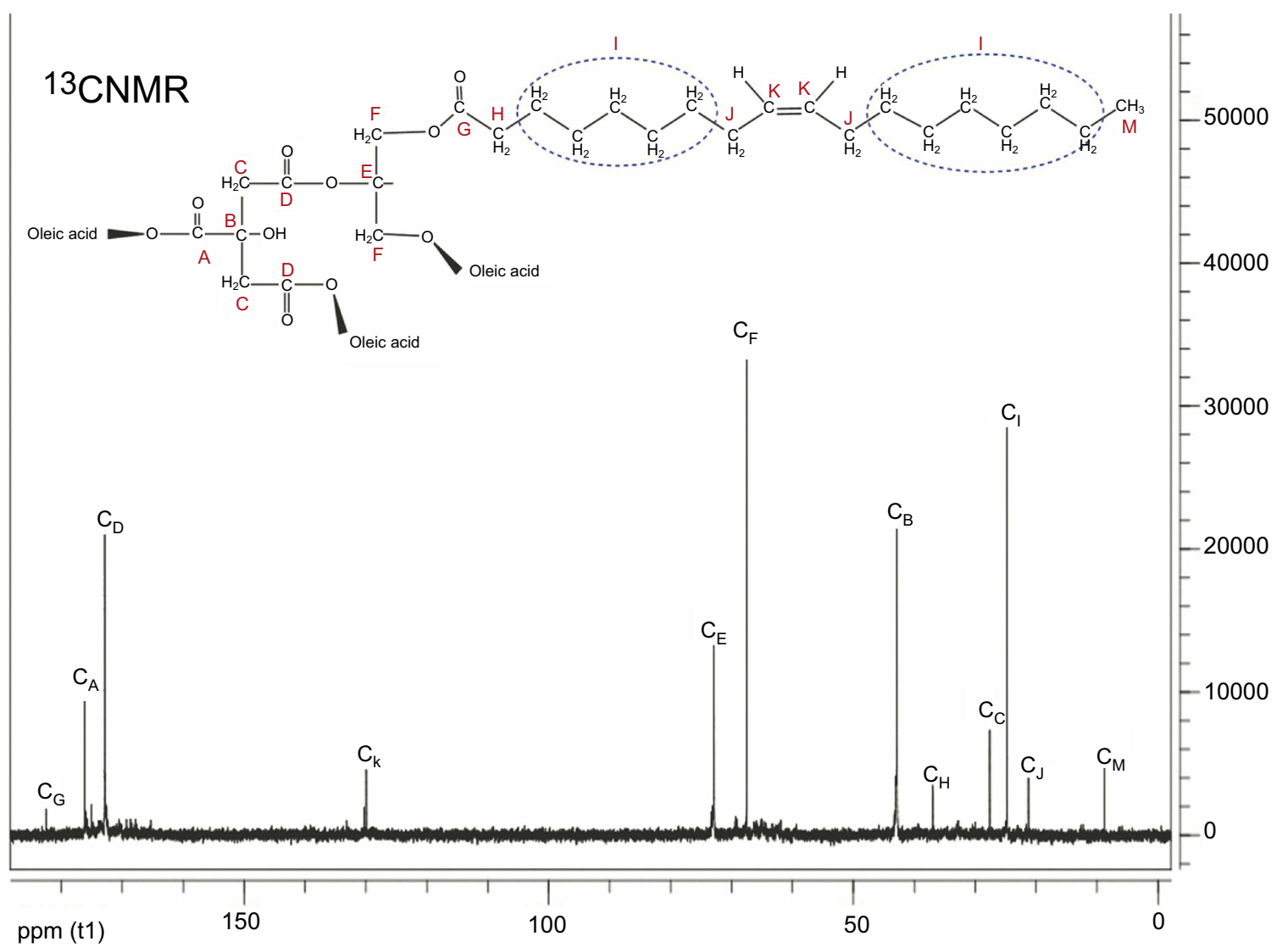

Figure $3{ }^{13} \mathrm{CNMR}$ spectrum of polymeric nanoparticle modified by oleic acid (NPMO) in D2O.

obtained diagram can be expressed by the purity of the resulting sample.

By using the DLS diagram (Figure 5), the size of the NPMO is monitored in water at $25^{\circ} \mathrm{C}$. The average shape shows the NPMO diameter based on intensity. The peak width of the courtyard is indicative of the fact that there is also a particle size sample solution larger than $200 \mathrm{~nm}$, but the diameter of most particles is estimated to be about 200 $\mathrm{nm}\left(\mathrm{PDI}=[\{402.768 / 200\}]^{\wedge} 2=4.05\right)$ according to the chart, which is a good measure for drug delivery.

The AFM results allowed morphology observation of the NPMO at two magnifications at the micro level (Figure 6). Submicrometer sizes of the NPMO are shown in Figure 6A-C. As is seen in Figure 6C, one crystal was chosen, its length and width with cross-sectional profile is shown in Figure 6. In the cross-sectional profile along the length and width of the crystal, interchange of heterogeneous wrinkles is visible. ${ }^{46}$

\section{Extraction and identification}

Based on the HPLC chromatogram, the standard Thymol broad peak was obtained at a retention time at 5.333 mins at a wavelength of $284 \mathrm{~nm}$. Thymol in the ethanolic extract of the Thymbra spicata, in contrast to standard Thymol, was in the same condition with a retention time of 5.233 mins (Figure 7A and B). Figure 7 shows that the well-separated peak of Thymol in Thymbra spicata extract was compared to standard Thymol, upon application of the developed method. The quantitative analysis revealed that Thymol was found to be predominant per ethanol fraction (18.64 $\mathrm{mg} / \mathrm{g}$ Thymol) of Thymbra spicata extract. The assay method was validated and the calibration curve was linear:

\section{Evaluation of the load and releasing}

By HPLC, loading capacity and release of free Thy and Thy in the E were evaluated from the NPMO. The amount of Thy and E loaded on NPMO was calculated using the standard Thymol-free curve. After three repetitions and injection into HPLC, the loading rate of Thy and $\mathrm{E}$ was estimated at $43 \pm 2.5 \%$ and $41 \pm 1.8 \%$, respectively.

Free Thymol and Thymol in the extract were released in two fast and slow phases of the NPMO. In the first $12 \mathrm{hrs}$, approximately $65 \%$ of loaded Thy and $62 \%$ of loaded E were released from a fast gradient of ThyNPMO and 


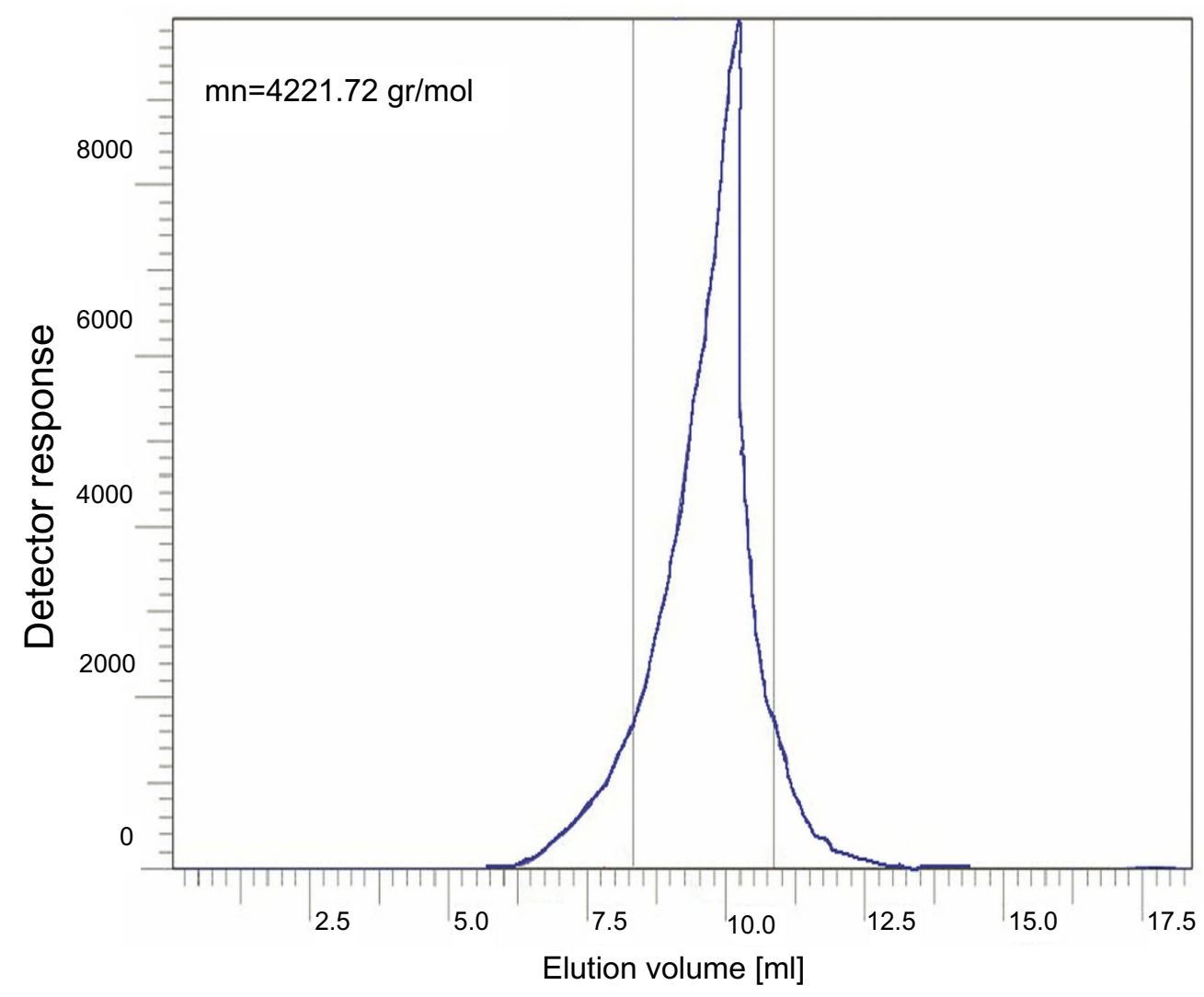

Figure 4 Gel permeation chromatography image of polymeric nanoparticles modified by oleic acid (NPMO).

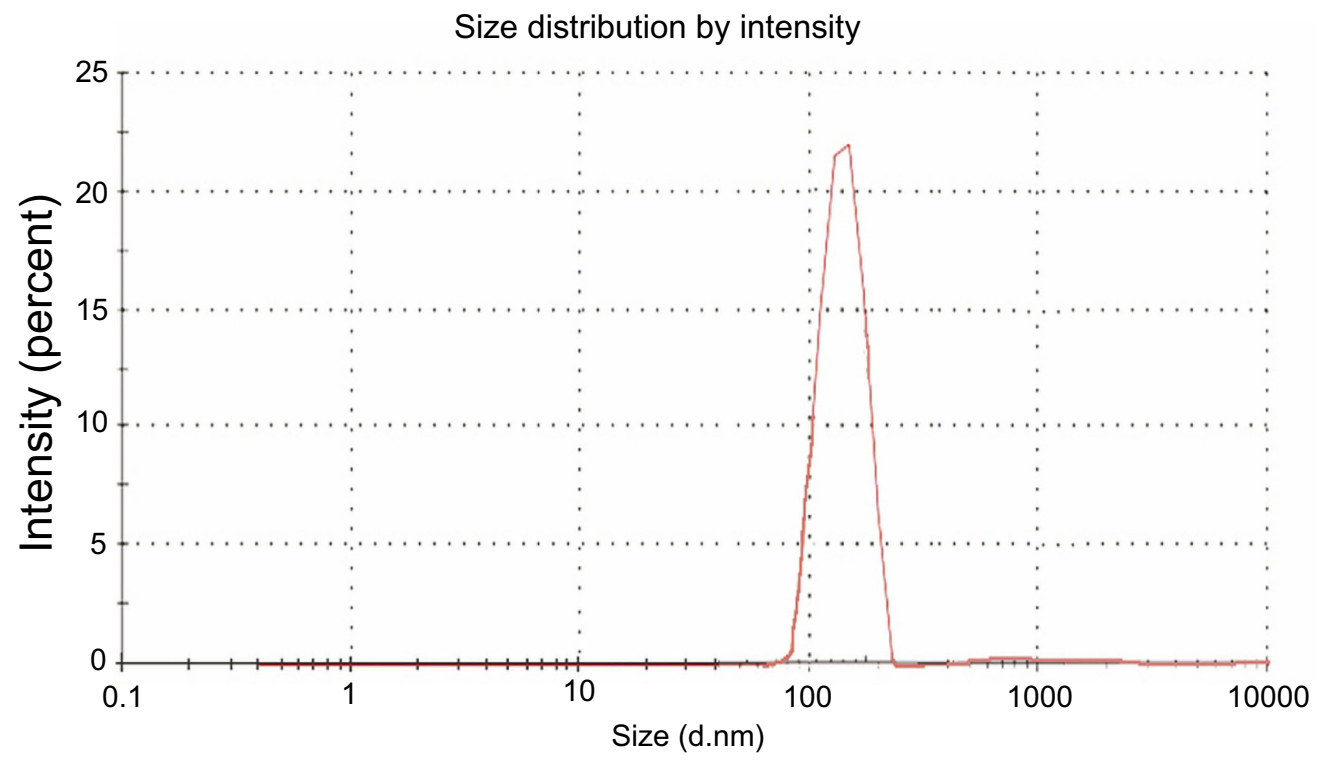

Figure 5 Dynamic light scattering diagram of polymeric nanoparticles modified by oleic acid (NPMO).

ENPMO, and the remaining $80-85 \%$ to $48 \mathrm{hrs}$ were slow biphasic kinetics with a faster release phase during the released at a lower rate and less slope. NPMO showed a initial period as shown in Figure 8. 


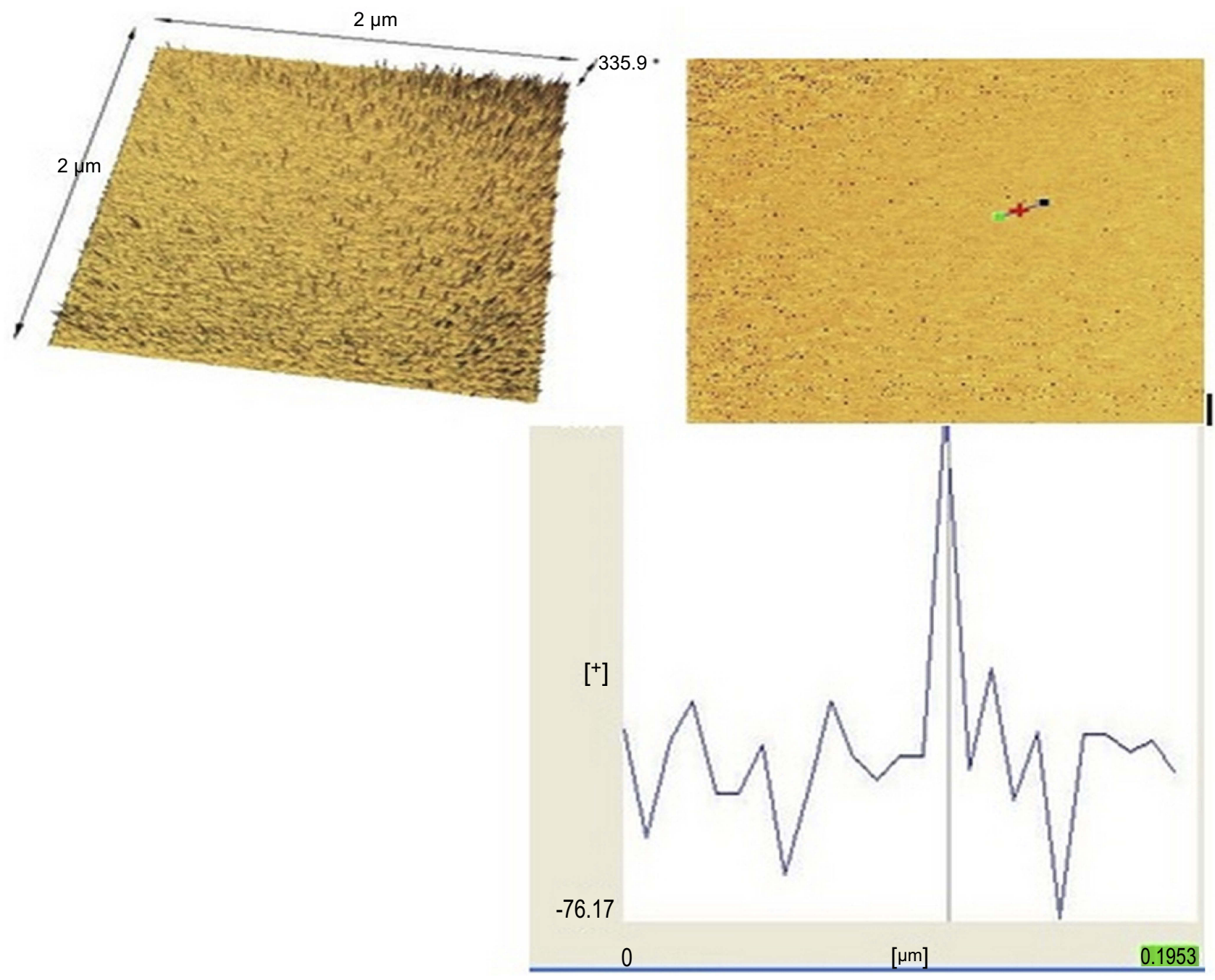

Figure 6 Atomic force microscopy (AFM) images of the nanoparticles modified by oleic acid (NPMO) surface morphology (top and bottom, topographical view); AFM image with the prior preparation of mounting samples.

\section{Thy, ThyNPMO, E, and ENPMO effects on cell viability}

After incubating of OECs with $\mathrm{NG}, \mathrm{HG}$, and $\mathrm{HM}$ at different times $(24,48$, and $72 \mathrm{hrs})$, cell viability significantly decreased in HG conditions in 48 hrs $(p<0.001$ vs NG control). Thy, ThyNPMO, E, and ENPMO at a low concentration, ie, 5, 10, 20, and $40 \mu \mathrm{M}$ (EC50 33 $\pm 1.5,22$ $\pm 0.9,35 \pm 1.8$, and $25 \pm 1.1 \mu \mathrm{M}$, respectively) ( $p<0.001$ vs HG control) prevented cell death from HG.

However, high concentrations, ie, 80, 120, and 180 $\mu \mathrm{M}$, led to a reduction in cell viability with LC50 values of $121 \pm 5.3,100 \pm 4.2,125 \pm 5.8$, and $106 \pm 5.3 \mu \mathrm{M}$, respectively in NG and $105 \pm 3.5,82 \pm 2.8,109 \pm 4.3$, and $86 \pm 3.4$ $\mu \mathrm{M}$, respectively in HG states in OECs. There was no evidence of cell death of Thy, ThyNPMO, E, and ENPMO at low concentrations in NG conditions, but at high concentrations, cell viability reduced in OECs ( $p<0.01$ vs NG, $p<0.001$ vs HG) (Figure 9). Molecularlevel experiments were limited to 20 and $40 \mu \mathrm{M}$ as low and 80 and $120 \mu \mathrm{M}$ as high Thy, ThyNPMO, E, and ENPMO concentrations which showed cytoprotective and cytotoxic activities, respectively.

\section{Effect of Thy, ThyNPMO, E, and ENPMO on ROS generation}

The formation of ROS in HG conditions significantly increased ( $p<0.001$ vs NG). Low concentrations of Thy, ThyNPMO, E, and ENPMO have prevented the formation of the ROS from HG conditions in the OECs. However, Thy, ThyNPMO, E, and E-NPMO at high concentrations increased the production of ROS in both HG and NG conditions. Mannitol did not alter intracellular ROS (Figure 10). 

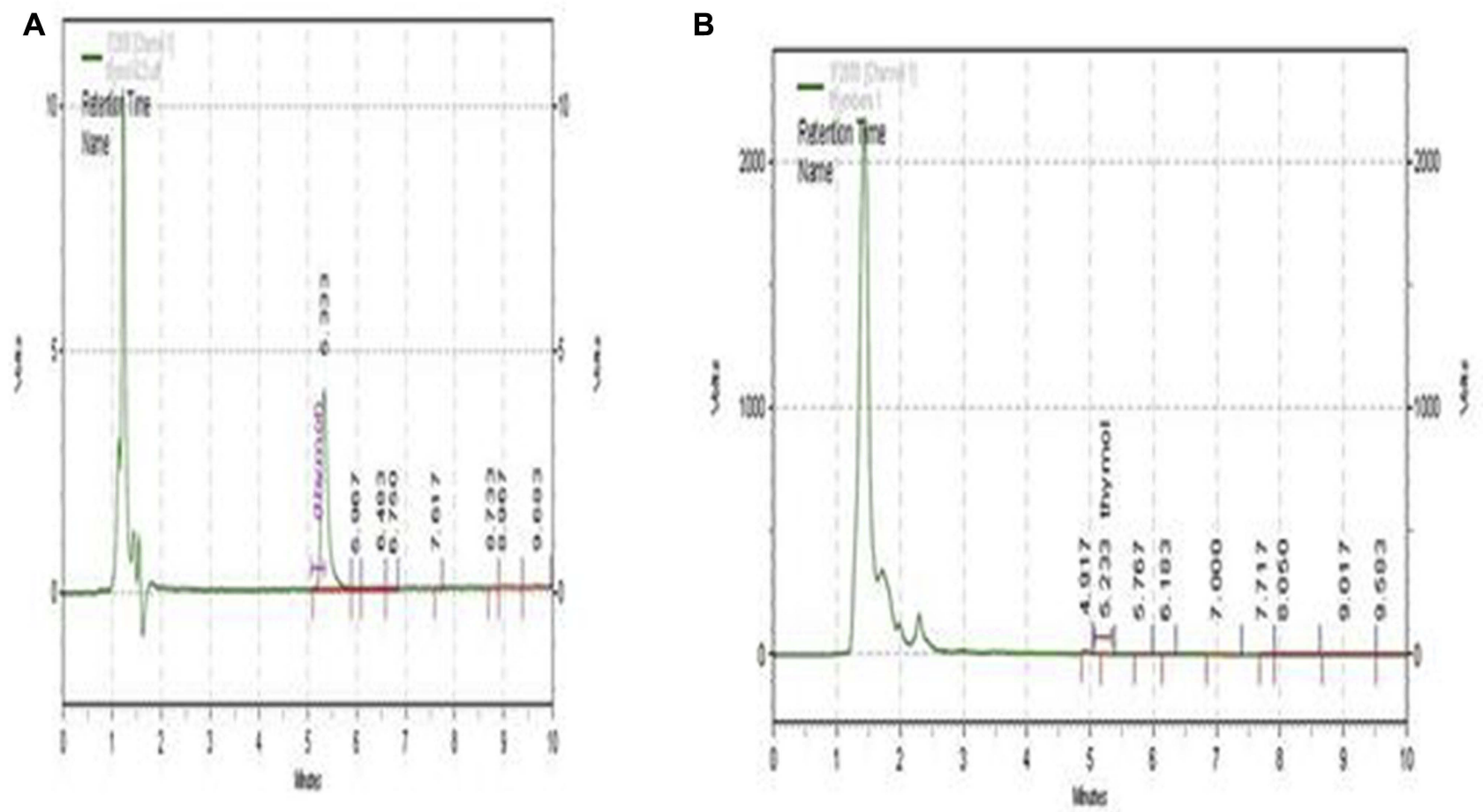

Figure 7 HPLC chromatogram of (A) Thymol standard and (B) Thymbra spicata extract. HPLC conditions were same for both Thymol and Thymbra spicata extract. Abbreviation: HPLC, high performance liquid chromatography.

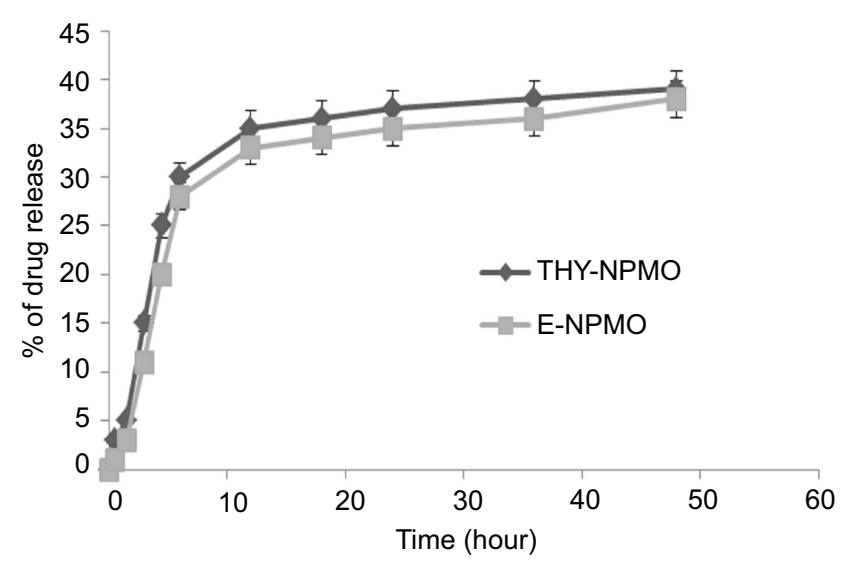

Figure 8 In vitro release of free Thy and Thy in E loaded nanoparticles modified by oleic acid (NPMO) $\left(0.1 \mathrm{~g}, 1.67 \times 10^{-2} \mathrm{mM}\right)$ in distilled water (HPLC grade) at $37^{\circ} \mathrm{C}$. Data represented as mean \pm SEM $(n=3)$.

\section{Effect of Thy, ThyNPMO, E, and ENPMO on $\mathrm{NO}$ production}

The production and release of $\mathrm{NO}$ in $\mathrm{HG}$ conditions increased compared to NG ( $p<0.001$ vs NG). Incubating OECs, with low concentrations of Thy, ThyNPMO, E, and ENPMO prevented the release and production of NO in HG conditions. However, at high concentrations, nitric oxide increased in both under NG and HG conditions. Incubation of OECs with L-NAME (1 mM) inhibited NO release under our experimental conditions (Figure 11).

\section{Effect of Thy, ThyNPMO, E, and ENPMO on ILK protein expression}

The HG condition in the OECs for $48 \mathrm{hrs}$ increased the ILK protein expression. This situation was also seen in mannitol (Figure 12). Low concentrations of Thy and $\mathrm{E}$ maintained ILK overexpression caused by HG whereas at high concentrations decreased ILK expression in both NG $(p<0.01-0.001$ vs NG control) and HG states $(p<0.001$ vs HG control).

\section{Effect of Thy, ThyNPMO, E, and ENPMO on VEGF protein expression}

The HG in the OECs increased the VEGF protein expression $(p<0.001 \mathrm{vs} \mathrm{NG})$ whereas mannitol did not affect the expression of VEGF. Treatment with low concentrations of Thy, ThyNPMO, E, and ENPMO decreased VEGF protein expres-

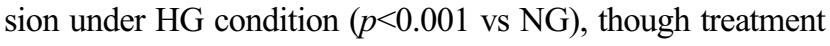
with high concentrations caused an increase in VEGF protein expression in both NG and HG states (Figure 13).

\section{Effect of Thy, ThyNPMO, E, and ENPMO on BDNF protein expression}

The BDNF protein expression decreased in OECs in $\mathrm{HG}$ state $(p<0.001$ vs NG) whereas mannitol did not affect. Treatment with low concentrations of Thy, ThyNPMO, E, 


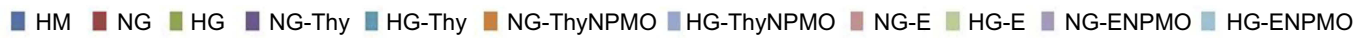

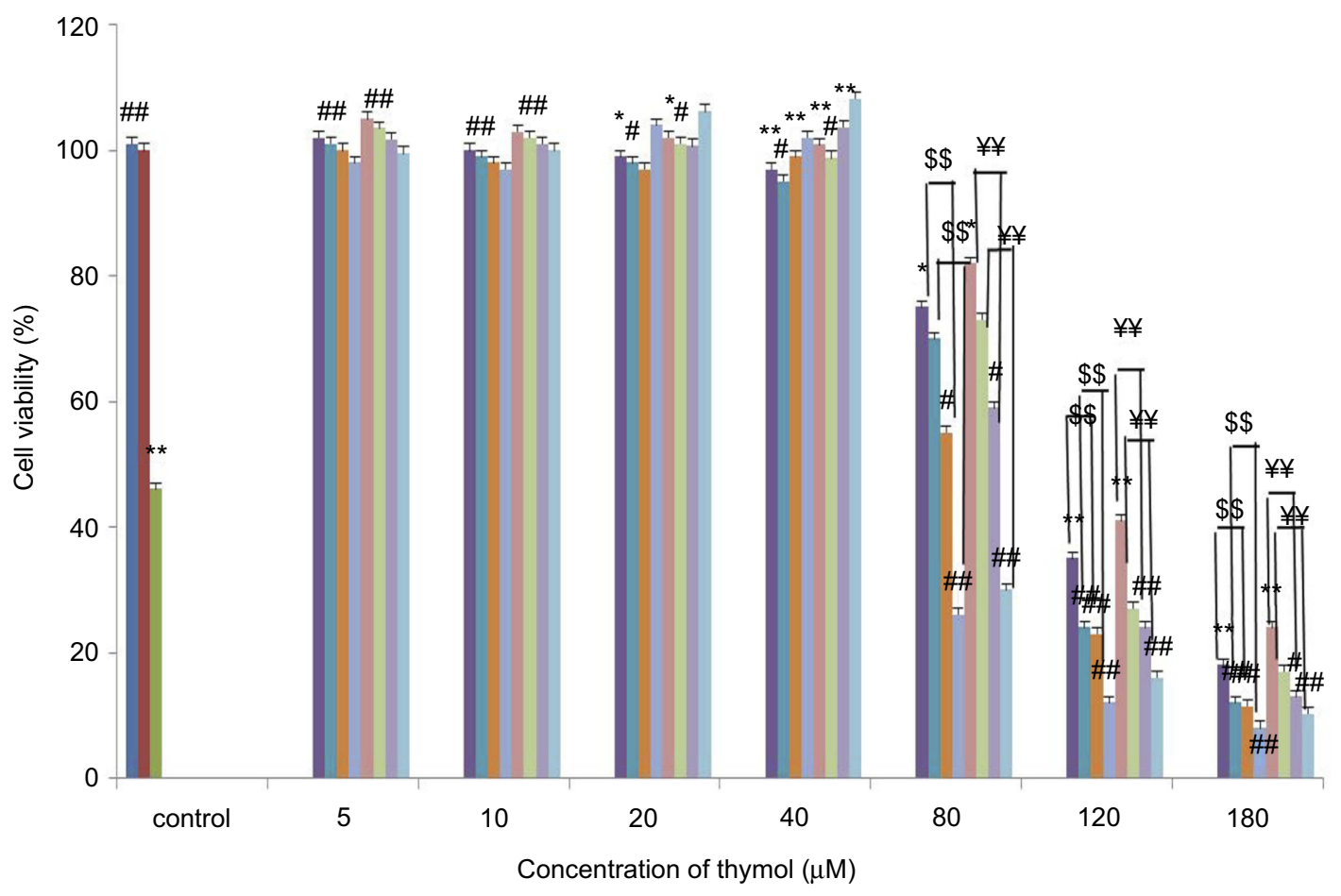

Figure 9 Effect of Thy, ThyNPMO, E, and ENPMO on olfactory ensheathing cells (OECs) cell viability. OECs were treated with normal glucose (NG), high glucose (HG), high mannitol (HM), NG plus Thymol (NG-Thy), HG plus Thymol (HG-Thy), NG plus Thymol polymeric nanoparticles modified by oleic acid (NG-ThyNPMO), HG plus Thymol polymeric nanoparticles modified by oleic acid (HG-ThyNPMO), NG plus extract (NG-E), HG plus extract (HG-E), NG plus extract polymeric nanoparticles modified by oleic acid (NG-ENPMO), HG plus extract polymeric nanoparticles modified by oleic acid (HG-ENPMO) at $37^{\circ} \mathrm{C}$ in $5 \% \mathrm{CO} 2 . \mathrm{Data}$ are expressed as mean \pm SEM.

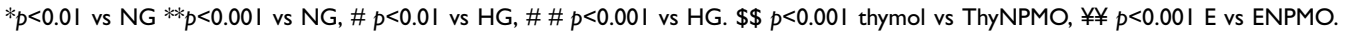

and ENPMO caused an increase in BDNF expression under HG state $(p<0.001$ vs NG), whereas treatment with Thy, ThyNPMO, E, and ENPMO on high concentrations caused a decrease in BDNF expression in both NG and HG states (Figure 14).

\section{Effect of Thy, ThyNPMO, E, and ENPMO on NGF protein expression}

The NGF protein expression decreased in OECs under HG state $(p<0.001$ vs NG) whereas mannitol did not affect. Treatment with low concentrations of Thy, ThyNPMO, E, and ENPMO caused an increase in NGF expression under HG state $(p<0.001$ vs NG), whereas treatment with Thy, ThyNPMO, E, and ENPMO on high concentrations caused a decrease in NGF expression in both $\mathrm{NG}$ and $\mathrm{HG}$ states (Figure 15).

\section{Discussion}

To reduce the side effects and reduction of dosing intervals, researchers are now focusing on drug delivery and design systems. In this regard, some components of medicinal plants such as Thymol, with a terpenoids structure, are used for a variety of disorders and diseases. ${ }^{47}$ However, the purpose of this study was to synthesize and evaluate the neurotrophic effects of Thy, ThyNPMO, E, and ENPMO in OECs in normal and HG (glucotoxicity) and their possible mechanisms.

Various NPs, such as polymeric, ceramic, metallic based ones, micelles, liposomes, carbon nanotubes, and dendrimers have been used for a variety of research. ${ }^{48}$ Recent research has focused on the possibility of using hyperbranched and functionalized polymers to enhance the efficiency of drug delivery systems due to their specific characteristics, such as low viscosity, high solubility, plenty of terminal groups, and lack of chain entanglements. ${ }^{49,50}$ Hyperbranched polymers have a similar configuration to dendrimers in terms of apparent structure, but they are more easily synthesized. ${ }^{51-53}$ In addition, to arrange their solubility, compatibility, reactivity, adhesivibility to specific surfaces, self-assimilability, and chemical recognizability, end-groups of hyperbranched polymers can be easily modified. ${ }^{54}$ It has also been documented 


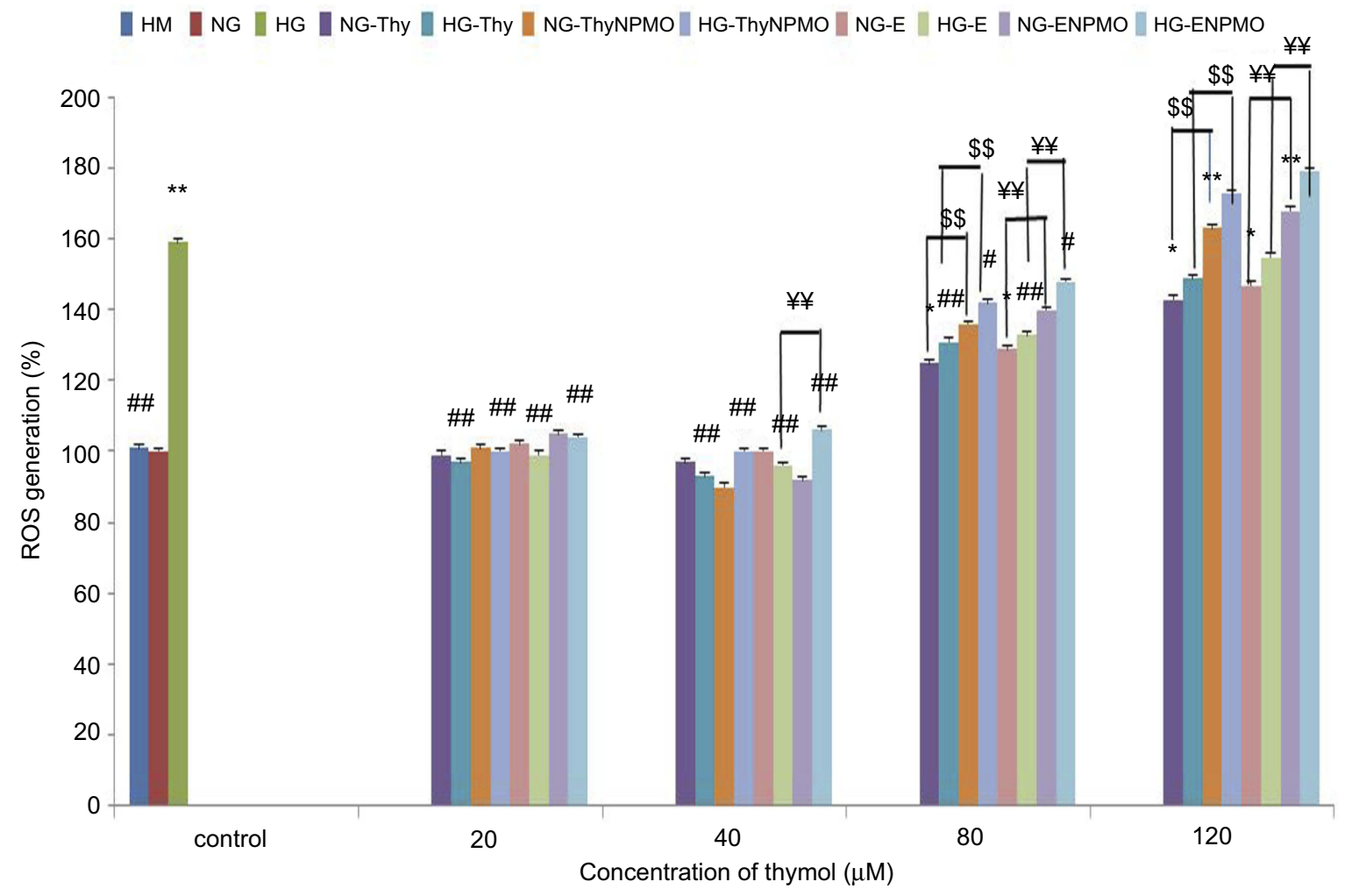

Figure 10 Effect of Thy, ThyNPMO, E, and E-NPMO on ROS generation in NG and HG states. Bar graphs showing ROS level (\%) in olfactory ensheathing cells (OECs) exposed to normal glucose (NG), high glucose (HG), high mannitol (HM), NG plus Thymol (NG-Thy), HG plus Thymol (HG-Thy), NG plus Thymol polymeric nanoparticles modified by oleic acid (NG-ThyNPMO), HG plus Thymol polymeric nanoparticles modified by oleic acid (HG-ThyNPMO), NG plus extract (NG-E), HG plus extract (HG-E), NG plus extract polymeric nanoparticles modified by oleic acid (NG-ENPMO), HG plus extract polymeric nanoparticles modified by oleic acid (HG-ENPMO). Data are expressed as mean \pm SEM. ${ }^{*} p<0.01$ vs NG and HM, ${ }^{* *} p<0.001$ vs NG and HM, \#\# $p<0.001$ vs $\mathrm{HG}$. $\$ \$ p<0.001$ thymol vs Thy NPMO, $¥ ¥ p<0.00$ I E vs ENPMO.

that hydrophilic poly (ethylene glycol) capped poly (lacticco-glycolic) acid NPs play an important role in glucose balance through the release of insulin-loaded. ${ }^{55}$ According to our findings, NPMO produced by thermal polycondensation has a good ability to carry material due to its biocompatibility. ${ }^{37}$ Due to factors such as small size and excellent biocompatibility, NPMOs are easily fluid in the bloodstream. Therefore, they will reach target tissues as well as increase the likelihood of binding to cell receptors. ${ }^{56}$ In this study, NPMO was synthesized with high water solubility and biocompatibility that use as promising materials for biomedical applications. This NPMO has some cavities for loading drugs and it was used for loading Thy and extract as a diabetes drug release system. It seems that all interactions between Thymol and NPMO were noncovalent. It has been investigated that NPMO can penetrate the cell through various mechanisms, including direct diffusion from the plasmid membrane or through receptors. ${ }^{57,58}$ In an investigation of NPs, citric glycerol hyperbranched polyester was synthesized in different concentrations that were monitored cisplatin as an anticancer drug for loading in polyester. ${ }^{20}$
In this study, different analytical techniques have been used to get NPMO structure, including FTIR, ${ }^{1} \mathrm{HNMR}$ and ${ }^{13}$ CNMR, DLS, GPC, and AFM. The FTIR spectrum demonstrated in polymeric NP, carbonyl ester group at $1734 \mathrm{~cm}^{-1}$, hydroxyl groups at $3375 \mathrm{~cm}^{-1}$, and for NPMO carbon derived from the carbonyl group at 1737 $\mathrm{cm}^{-1}$ and hydroxyl group at $3485 \mathrm{~cm}^{-1}$. The ${ }^{1} \mathrm{HNMR}$ spectrum showed that there are seven types of hydrogens. Hydrogen A is at $4.37 \mathrm{ppm}$. Hydrogen B is at $3.87 \mathrm{ppm}$. Hydrogen $\mathrm{C}$ is a methylated glycerol, which has two separate couriers due to the presence of neighbor hydrogen. Hydrogen D is at $2.45 \mathrm{ppm}$ due to the presence of two hydrogen neighbors. Hydrogen $\mathrm{E}$ is a methyl group adjacent to hydrogen D. Hydrogen F appears in a single pixel at $2 \mathrm{ppm}$ because of the same space-spatial position. Hydrogen I has a peak in the range of $2.2-2.3 \mathrm{ppm}$. The G-hydrides appear to be in the form of a double peak at $5.26 \mathrm{ppm}$. Hydrogen $\mathrm{J}$ is a multiplicative peak at $1.15 \mathrm{ppm}$ appear. The ${ }^{13} \mathrm{CNMR}$ spectrum of the NPMO compound displays 12 types of carbon in the synthetic composition. Carbon A is displacement of $177 \mathrm{ppm}$ and carbon B is at 


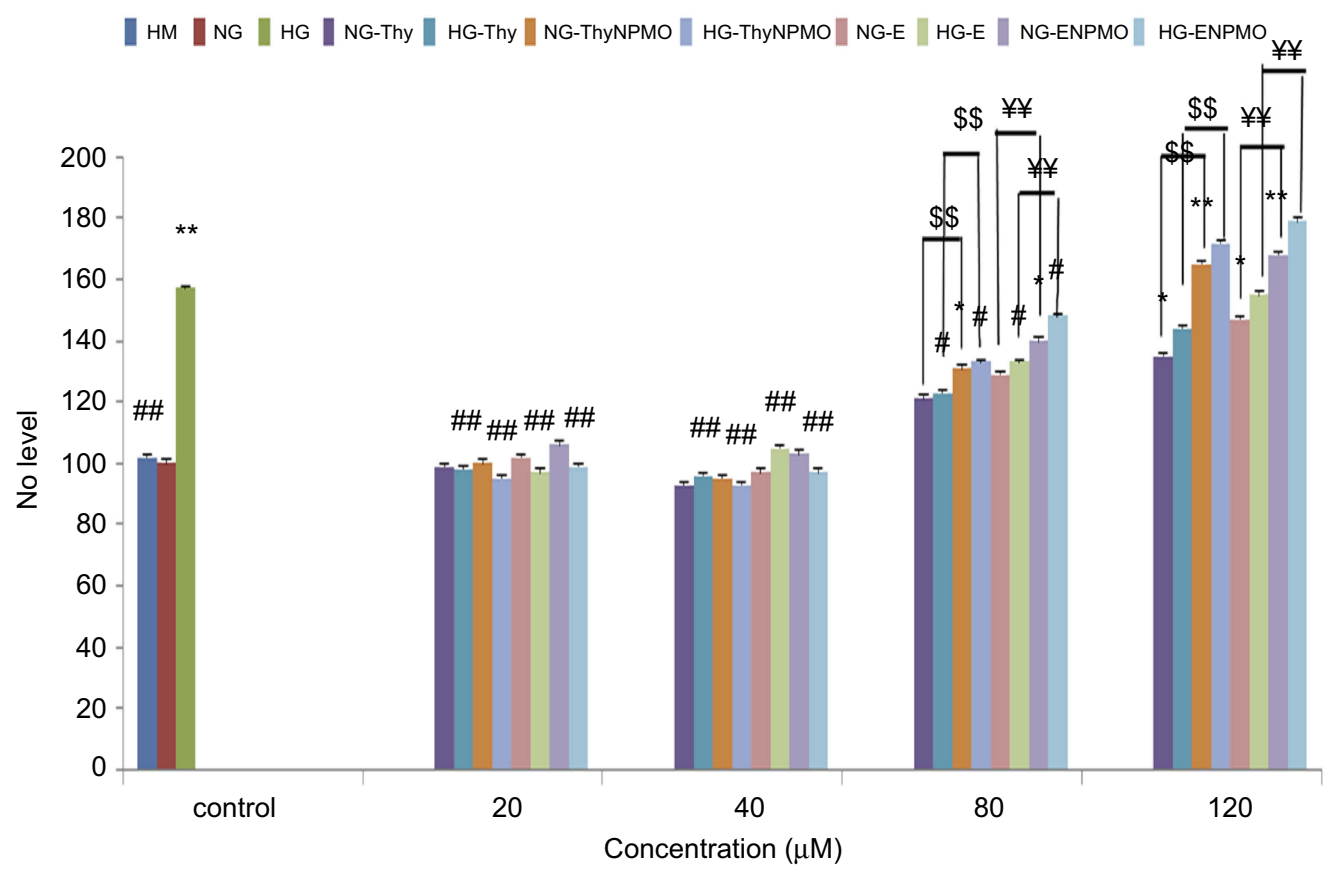

Figure I I Effect of Thy, ThyNPMO, E, and ENPMO on nitric oxide (NO) production in NG and HG states. Bar graphs showing NO level (\%) in olfactory ensheathing cells (OECs) exposed to normal glucose (NG), high glucose (HG), high mannitol (HM), NG plus Thymol (NG-Thy), HG plus Thymol (HG-Thy), NG plus Thymol polymeric nanoparticles modified by oleic acid (NG-ThyNPMO), HG plus Thymol polymeric nanoparticles modified by oleic acid (HG-ThyNPMO), NG plus extract (NG-E), HG plus Extract (HG-E), NG plus extract polymeric nanoparticles modified by oleic acid (NG-ENPMO), HG plus extract polymeric nanoparticles modified by oleic acid (HG-

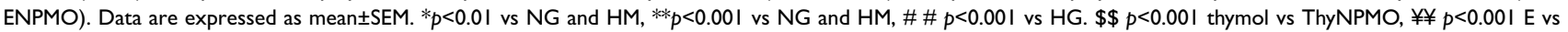
ENPMO.

42 ppm. Methionine CA carbonated with the letter C appears at $28 \mathrm{ppm}$ and carbon D appears at $172 \mathrm{ppm}$. Carbon $\mathrm{E}$ is given at $73 \mathrm{ppm}$ and $\mathrm{F}$, which appears as a peak at the highest intensity of $68 \mathrm{ppm}$. Carbon $\mathrm{G}$ has the largest displacement and peak at 183 ppm. Carbon $\mathrm{H}$ marked with a peak of $38 \mathrm{ppm}$ and "I" has a roughly identical position at $26 \mathrm{ppm}$. The adjacent dual-carbon carbons $\mathrm{J}$ have a peak at $21 \mathrm{ppm}$ and $\mathrm{K}$ appears as a peak at $130 \mathrm{ppm}$. At the end of the carbon, the methyl group has appeared with a minimum displacement of 9 ppm. The obtained molecular weight (GPC technique) for copolymer was around $4221.72 \mathrm{~g} / \mathrm{mol}$. According to the DLS tests, hydrodynamic diameter of hyperbranched polyester is around $200 \mathrm{~nm}$. With regard to the results of AFM, it can be concluded that NPMO is a natural microand mesoporous material with polymodal pore size distribution. The microporosity is connected with the NPMO, while the mesoporosity is caused by cleavage phenomenon of the NPMO.

By HPLC method, it is possible to evaluate the amount of NPMO loading capacity. In this analysis, subsequent to drawing standard curve amount of the loading rate of Thy and Extract was estimated at $43 \pm 2.5 \%$ and $41 \pm 1.8 \%$, respectively. In one study, chitosan NP of thymol with anti-bacterial effects had a loading capacity of about $2.5 \% .{ }^{59}$ However, in another study, Thymol nanospheres as an effective anti-bacterial agent, similar to that of our study, was reported to be about $43 \%{ }^{24}$

It is important to note that the release pattern of drug from NPs and the duration of exposure to cells, as a drug release system, can have higher efficacy and safety. ${ }^{60}$ In our study, approximately $65 \%$ of Thymol and $62 \%$ of extract were released from the NPMO in the first $12 \mathrm{hrs}$ (fast phase) and the remaining $80-85 \%$ in the next 48 hrs was released with slower kinetics and less slope, which indicated two phases the release of Thymol from NPMO. There are several mechanisms, including surface erosion, disintegration, diffusion and desorption for drug or essential oil release from NP and microparticles. ${ }^{61}$ The initial and rapid release of the drug from the polymer is more closely related to the particles of the drug that are surface-coated with polymeric NPs, and thus the release of the drug increases. ${ }^{62}$ Due to the drug absorbed on the NPMO's surface, Thymol was already quantified in the first hours of experiment and it also called the burst effect. ${ }^{63,64}$ In a study, it has been shown that Thymol has released about $60 \%$ of NPs from natural lipids for up to $18 \mathrm{hrs} .{ }^{65}$ In another study, it has been shown that Thymol loaded with hierarchically structured biogenic silica in the first 48 hrs has been released about $50 \%$ of 


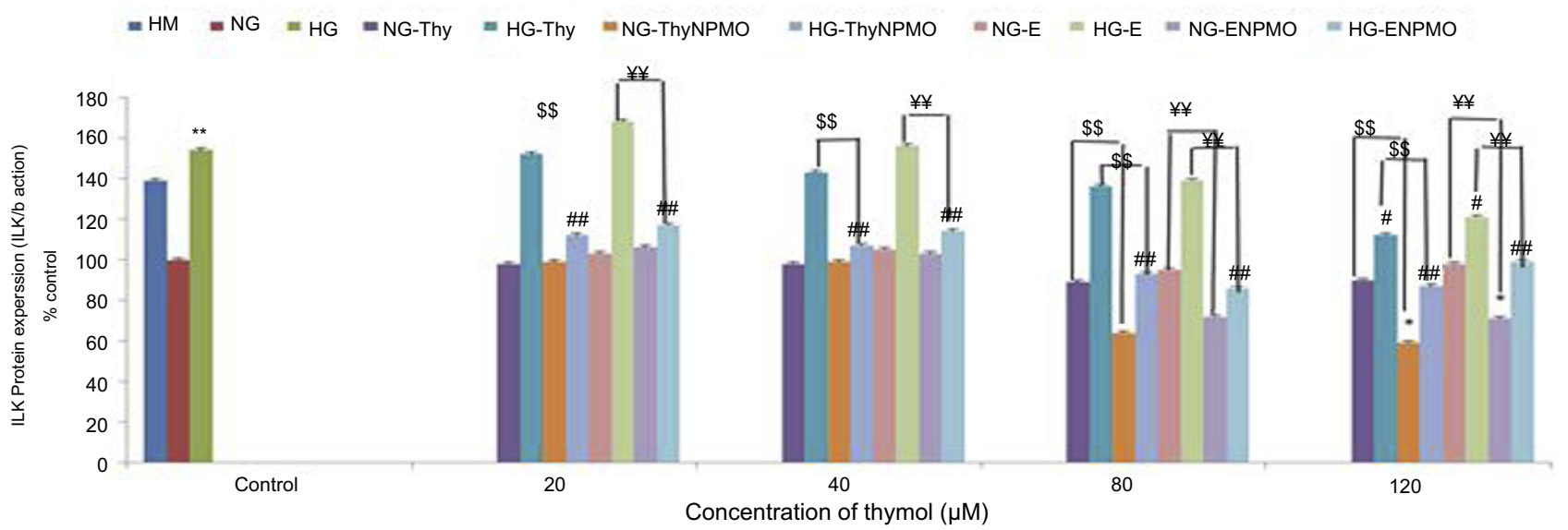

HM
$N P$
$N G$
HG
NG NP S
NG NP S
NG EX
HG EX
NG NP EX
HG NP EX
B actin

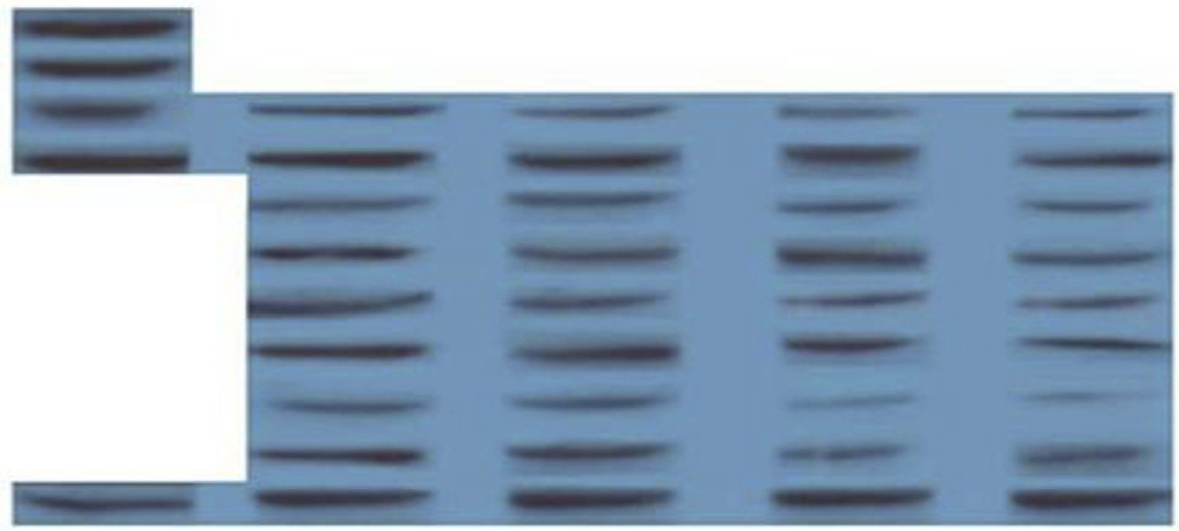

Figure 12 Effect of Thy, ThyNPMO, E, and ENPMO on ILK in OECs in normal glucose (NG) and high glucose (HG) states. Protein expression of ILK (\%) in olfactory ensheathing cells (OECs) exposed to normal glucose (NG), high glucose (HG), high mannitol (HM), NG plus Thymol (NG-Thy), HG plus Thymol (HG-Thy), NG plus Thymol polymeric nanoparticles modified by oleic acid (NG-ThyNPMO), HG plus Thymol polymeric nanoparticles modified by oleic acid (HG-ThyNPMO), NG plus extract (NG-E), HG plus extract (HG-E), NG plus extract polymeric nanoparticles modified by oleic acid (NG-ENPMO), HG plus extract polymeric nanoparticles modified by oleic acid (HG-ENPMO). Data are expressed as mean \pm SEM. ${ }^{*} p<0.01$ vs NG and HM, ${ }^{* *} p<0.00$ I vs NG and HM, \#\# $p<0.00$ I vs $\mathrm{HG}$. $\$ \$ p<0.00 \mathrm{I}$ thymol vs Thy NPMO, $¥ p<0.00$ I E vs ENPMO.

Thymol. ${ }^{66}$ Numerous studies have reported that increased chronic blood glucose not only causes glucose metabolic disorders in the brain ${ }^{67}$ but also destroys the nervous system and impairment cognitive activity. ${ }^{68}$ Disease related to diabetes includes Alzheimer's disease, dementia, degradation of the peripheral nervous system, and neuronal disorders of the eye. ${ }^{69,70}$ Hyperglycemia by increasing the ROS causes neuropathy due to oxidative stress. Moreover, angiogenesis activity has also been shown to associate with an overexpression of a PI3 kinase-Akt-eNOS pathway and an increased VEGF production. ${ }^{71}$

In the present study, treatment with Thy, ThyNPMO, E, and E-NPMO at low concentrations $(5,10,20$, and $40 \mu \mathrm{M})$ prevented HG-mediated cell death with EC50 values of 33 $\pm 1.5,22 \pm 0.9,35 \pm 1.8$, and $25 \pm 1.1 \mu \mathrm{M}$, respectively and at high concentrations $(80,120$, and $180 \mu \mathrm{M})$ reduced cell viability with LC50 values of $121 \pm 5.3,100 \pm 4.2 \mu \mathrm{M}, 125 \pm 5.8$, and 106 \pm 5.3 , respectively in $\mathrm{NG}$ and $105 \pm 3.5,82 \pm 2.8,109 \pm 4.3$, and $86 \pm 3.4 \mu \mathrm{M}$, respectively in HG states in OECs. It seems that cytotoxicity of Thy, ThyNPMO, E, and E-NPMO is almost equipotent in either $\mathrm{NG}$ or $\mathrm{HG}$ conditions. It has already been observed that Thymol with EC $50=0.74 \mu \mathrm{M}$ has been used by inhibiting acetylcholine esterase in Alzheimer's disorders. ${ }^{72}$ Also, Thymol is used by EC50=12 $\mu \mathrm{M}$, due to its effect on cortical cells and the mechanism of GABA stimulation, for neurodegenerative disorders. ${ }^{73}$ But regarding toxic effects, it has been reported that Thymol triggered cytotoxicity inMCF-7 breast cancer cell lines with an LC50 of $2.5 \mathrm{mg} / \mathrm{mL}^{73}$ Moreover, it has been shown that the Ocimum kilimandsc haricum Leaf Extract Engineered Silver Nanoparticles which contains terpenoids-like Thymol structures reduce the cell viability of the Aedesaegypti with LC50 $=0.009 \mathrm{ppm}^{74}$ 


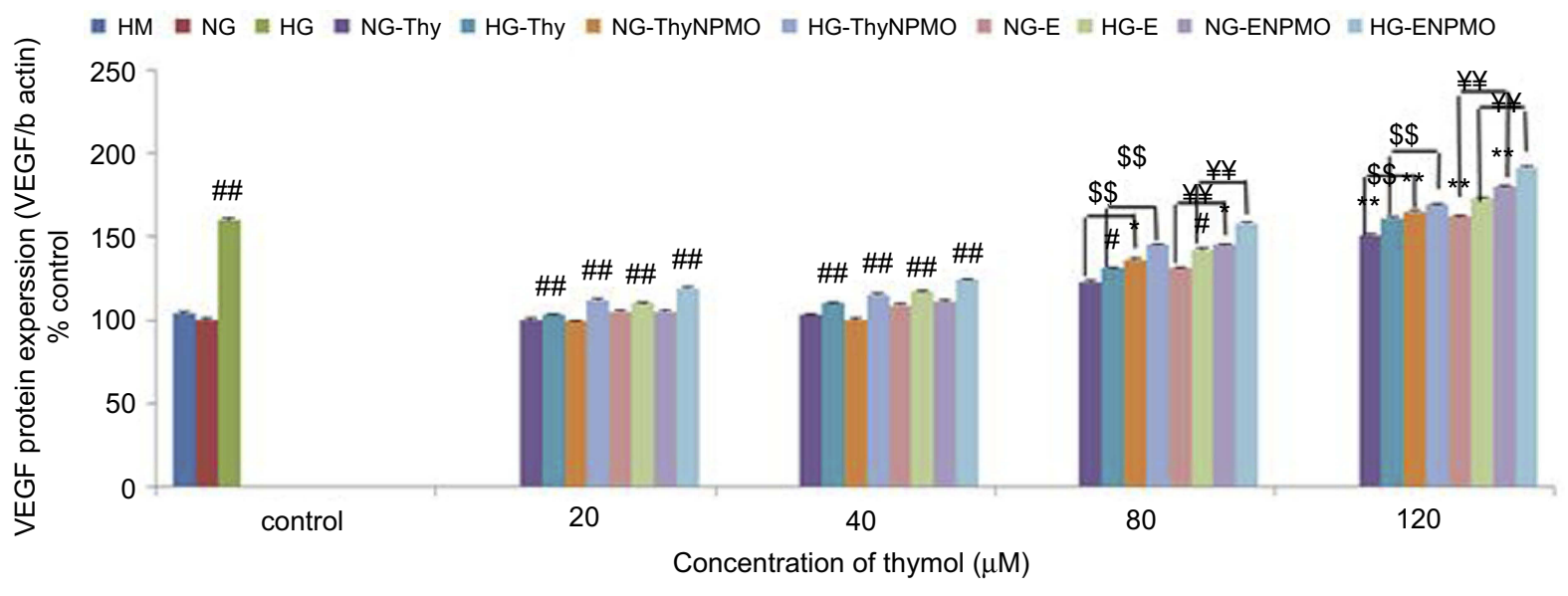

HM
$N P$
$N G$
$H G$
NG NP S
HG NP S
NG EX
HG EX
NG NP EX
HG NP EX
B actin

Figure 13 Effect of Thy, ThyNPMO, E, and ENPMO on VEGF in OECs cell in normal glucose (NG) and high glucose (HG) states. Protein expression of VEGF (\%) in olfactory ensheathing cells (OECs) exposed to normal glucose (NG), high glucose (HG), high mannitol (HM), NG plus Thymol (NG-Thy), HG plus Thymol (HG-Thy), NG plus Thymol polymeric nanoparticles modified by oleic acid (NG-ThyNPMO), HG plus Thymol polymeric nanoparticles modified by oleic acid (HG-ThyNPMO), NG plus extract (NG-E), HG plus extract (HG-E), NG plus extract polymeric nanoparticles modified by oleic acid (NG-ENPMO), HG plus extract polymeric nanoparticles modified by oleic acid (HG-ENPMO). Data are expressed as mean \pm SEM. ${ }^{*} p<0.01$ vs NG and HM, ${ }^{* *} p<0.00$ I vs NG and HM, \#\# $p<0.00$ I vs HG. $\$ \$ p<0.00$ I thymol vs ThyNPMO, $¥ ¥$ $p<0.001$ E vs ENPMO.

It has been investigated that $\mathrm{HG}(35 \mathrm{mM})$ induces hyperglycemic stress, ROS production ${ }^{75}$ and NO generation, injury of neuronal cells. ${ }^{76}$ Oxidative stress through three mechanisms effect on energy level and survival in the mitochondria of neuronal cells. First, in the presence of excess $\mathrm{O}^{2-} \mathrm{NO}$ is converted to ONOO- and in the following, ONOO- profoundly affects mitochondrial function and inhibits ATP synthesis. ${ }^{77,78}$ Second, mitochondrial oxidative stress through excess $\mathrm{O}^{2-}$. and $\mathrm{ONOO}-$ production inhibits the import of essential proteins to the mitochondria. ${ }^{79}$ Finally, oxidative damage of existing inner membrane proteins induces membrane permeability transition, a permeabilization of the mitochondrial inner membrane that causes apoptosis in cells. ${ }^{80}$

In the present study, HG significantly increased intracellular ROS formation. Incubation of the cells with low concentrations of Thy, ThyNPMO, E, and ENPMO and vitamin C $(100 \mathrm{mM})$ prevented this increase in fluorescence. However, high concentrations of Thy, ThyNPMO, E, and ENPMO increased ROS generation in both NG and HG states. NO release was significantly reduced in HG-cultured OECs. Treatment with low concentrations of Thy, ThyNPMO, E, and ENPMO restored NO release in OECs exposed to HG; however, NO release was reduced after treatment with high concentrations of Thy, ThyNPMO, E, and ENPMO in both NG- and HG-treated OECs. It has been shown that terpenoids, such as Thymol and carvacrol, have inhibitory effects on the central nervous system by inhibiting the release of NO in microglial cells. ${ }^{81}$ In addition, to the effects in NO, it has also been effective on intracellular reactive oxygen species in microglial cells in vitro. ${ }^{82}$

With the effect of Vernonia amygdalina that contains the structure of terpenoids on the cells of the rat brain tissue, it has been shown that in conditions of HG uptake in these cells, the amount of ROS increased, the amount of glutathione decreased, and thus the amount of NO also 


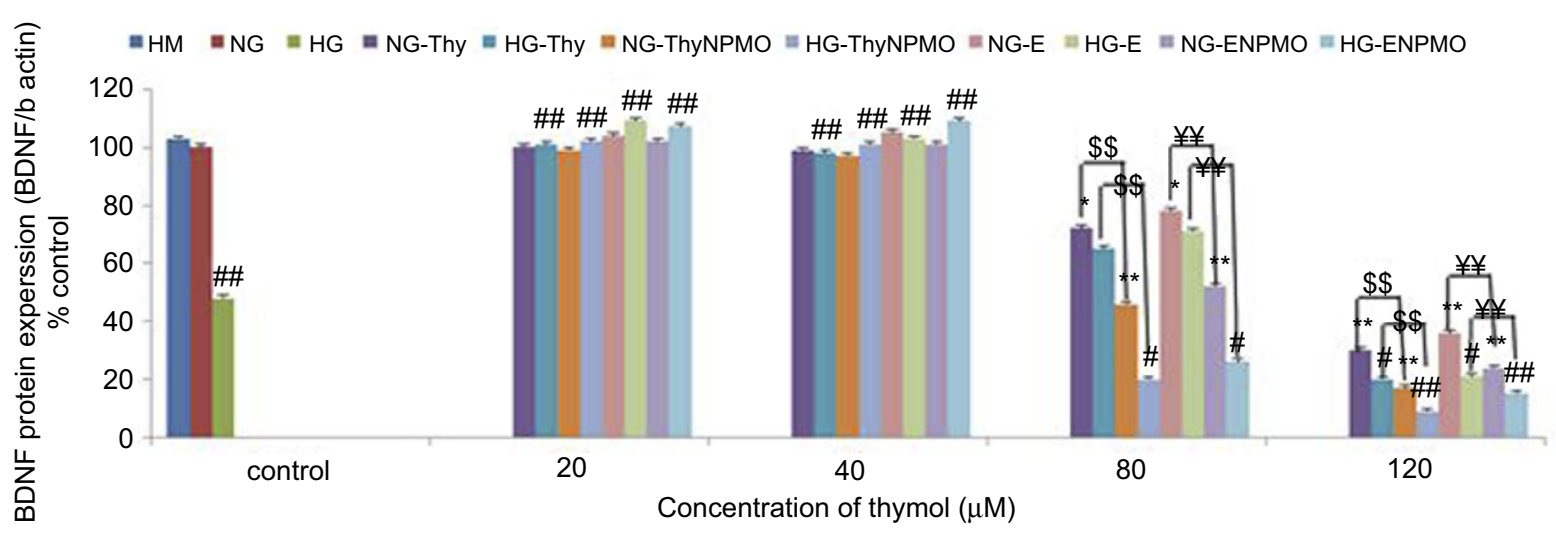

HM
NP
NG
HG
NG NP S
HG NP S
NG EX
HG EX
NG NP EX
HG NP EX
B actin

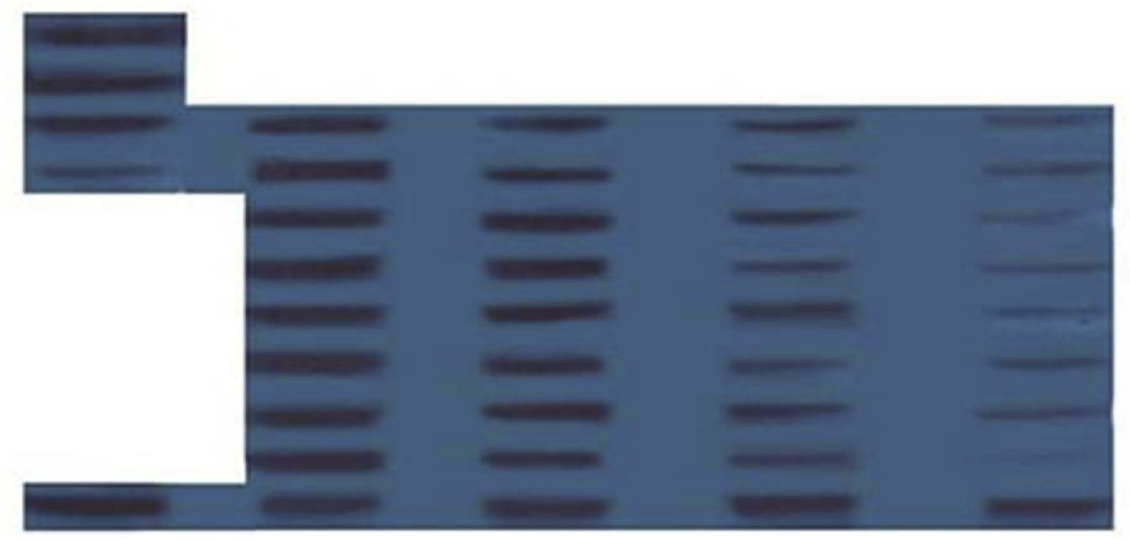

Figure 14 Effect of Thy, ThyNPMO, E, and ENPMO on BDNF in OECs in normal glucose (NG) and high glucose (HG) states. Protein expression of BDNF (\%) in olfactory ensheathing cells (OECs) exposed to normal glucose (NG), high glucose (HG), high mannitol (HM), normal glucose (NG), high glucose (HG), high mannitol (HM), NG plus Thymol (NG-Thy), HG plus Thymol (HG-Thy), NG plus Thymol polymeric nanoparticles modified by oleic acid (NG-ThyNPMO), HG plus Thymol polymeric nanoparticles modified by oleic acid (HG-ThyNPMO), NG plus extract (NG-E), HG plus extract (HG-E), NG plus extract polymeric nanoparticles modified by oleic acid (NG-ENPMO), HG plus extract polymeric nanoparticles modified by oleic acid (HG-ENPMO). Data are expressed as mean \pm SEM. $* p<0.01$ vs NG and HM, ** $p<0.00 \mathrm{I}$ vs NG and HM, \#\# $p<0.00$ I vs $H G, \$ \$ p<0.00$ I thymol vs ThyNPMO, $¥ p<0.00$ I E vs ENPMO.

changed. ${ }^{83}$ Therefore, increased antioxidant availability may be helpful in preventing or slowing the progress of various oxidative stress-related diseases. ${ }^{84}$

Endothelial ILK plays an important role in vascular endothelial cell growth. ${ }^{85}$ ILK expression has been reported to be rapidly controlled in diabetes, and for this reason, some authors find it effective in pathogenesis of diabetes mellitus. ${ }^{86}$

It has been reported that knocking down of ILK gene expression with siRNA inhibited the elevation of VEGF and ICAM 1. Also, it has been suggested that ILK has been involved in the response of cells to HG and may, therefore, play a role in the pathogenesis of diabetic retinopathy. ${ }^{87}$ It has been reported that terpenoid is a potent activator of Protein kinase C (PKC), and on the other hand, the synaptogenesis of astrocyte cells increases with integrin that acts through PKC. Thus, terpenoids may increase intragranularity. ${ }^{88}$ In the present study, we demonstrated that in the OECs, the HG condition increased the ILK protein expression. This situation was also seen in mannitol. Thy and $\mathrm{E}$, at low concentrations maintained ILK overexpression caused by $\mathrm{HG}$ whereas at high concentrations decreased ILK expression in both NG and HG states. In the following, our results showed that HG in the OECs increased the VEGF protein expression whereas mannitol did not affect. Treatment with low concentrations of Thy, ThyNPMO, E, and ENPMO decreased VEGF protein expression under HG state, though treatment with high concentrations caused an increase in VEGF protein expression in both NG and HG states. As well as, hyperglycemia effects can produce a lot of biological effects, such as increased expression of VEGF which is very crucial for the development of diabetic retinopathy. ${ }^{89}$ In a study using Thymol at 40 $\mathrm{mg} / \mathrm{kg}$ per day for 5 weeks, the activation of VEGF was significantly inhibited. ${ }^{90}$ Part of Thymol's protective 


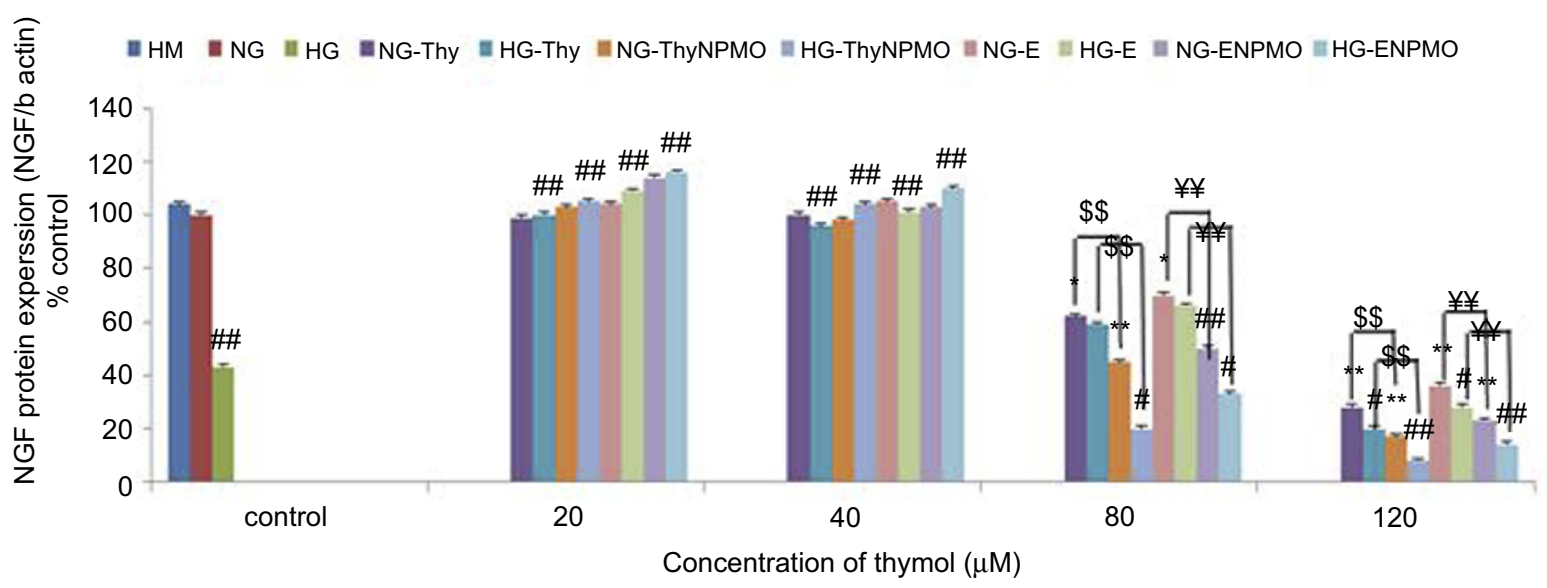

HM
NP
NG
HG
NG NP S
HG NP S
NG EX
HG EX
NG NP EX
HG NP EX
B actin

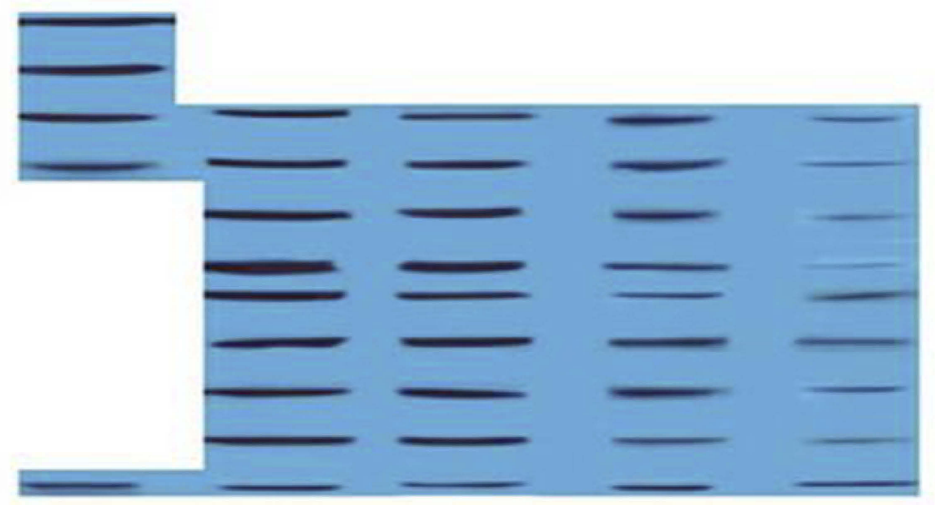

Figure 15 Effect of Thy, ThyNP, E, and ENP on NGF in OECs in normal glucose (NG) and high glucose (HG) states. Protein expression of NGF (\%) in olfactory ensheathing cells (OECs) exposed to normal glucose (NG), high glucose (HG), high mannitol (HM), normal glucose (NG), high glucose (HG), high mannitol (HM), NG plus Thymol (NGThy), HG plus Thymol (HG-Thy), NG plus Thymol polymeric nanoparticles modified by oleic acid (NG-ThyNPMO), HG plus Thymol polymeric nanoparticles modified by oleic acid (HG-ThyNPMO), NG plus extract (NG-E), HG plus extract (HG-E), NG plus extract polymeric nanoparticles modified by oleic acid (NG-ENPMO), HG plus extract polymeric nanoparticles modified by oleic acid (HG-ENPMO). Data are expressed as mean \pm SEM. ${ }^{*} p<0.01$ vs $N G$ and $\mathrm{HM}$, $* * p<0.00 \mathrm{I}$ vs $\mathrm{NG}$ and $\mathrm{HM}$, \# \# $p<0.00 \mathrm{I}$ vs HG. $\$ \$ p<0.00$ I thymol vs ThyNPMO, $¥ p<0.00$ I E vs ENPMO.

effect appears to be reduced to the expression of this protein.

It has been documented that HG level enhanced the protein expression of NGF and BDNF in Schwann cells in vitro. ${ }^{91}$ In the present study, we demonstrated that in OECs in HG state the BDNF and NGF protein expression decreased whereas mannitol did not affect. Treatment with low concentrations of Thy, ThyNPMO, E, and ENPMO caused an increase in BDNF and NGF expression under $\mathrm{HG}$ state, whereas treatment with Thy, ThyNPMO, E and ENPMO on high concentrations caused a decrease in BDNF expression in both NG and HG states. Similar to the protective status of this study, iridoid glycosides as a monoterpenoid agent was able to prevent the loss of the number of hippocampal cells by increasing the production of BDNF and NGF, as well as improving the memory status of the rat. ${ }^{92}$ Furthermore, the Curcuma longa L. (Curcumin 50, 100, or
$200 \mathrm{mg} / \mathrm{kg}$ ) containing the terpenoid structures has also been used to increase the expression of BDNF protein in the treatment of depression. ${ }^{93}$ It was recently found that cannabis extract, which is rich in terpenes and flavonoids, can increase the expression of NGF by enhancing the growth of the nerve in a rat model. ${ }^{94}$

\section{Abbreviation list}

HG, high glucose; NG, normal glucose; HM, high mannitol; Thy, Thymol; NPMO, polymeric nanoparticles modified by oleic acid; E, extract; OEC, olfactory ensheathing cells; G, glycerol; CA, citric acid; NMR, nuclear magnetic resonance; FTIR, Fourier-transform infrared; GPC, gel permeation chromatography; AFM, atomic force microscopy; DLS, dynamic light scattering; NO, nitric oxide; ILK, integrin-linked kinase; VEGF, vascular endothelial 
growth factor; BDNF, brain-derived neurotrophic factor; NGF, nerve growth factor.

\section{Acknowledgments}

This study was supported by Islamic Azad University, Ilam branch, Iran and Ilam University of Medical Sciences (grant no. 87-04-33-8073).

\section{Disclosure}

The authors report no conflicts of interest in this work.

\section{References}

1. Park W, Na K. Advances in the synthesis and application of nanoparticles for drug delivery. Wiley Interdiscip Rev Nanomed Nanobiotechnol. 2015;7:494-508. doi:10.1002/wnan.1325

2. Xia Y, Wu X, Zhao J, et al. Three dimensional plasmonic assemblies of AuNPs with an overall size of sub-200 nm for chemo-photothermal synergistic therapy of breast cancer. Nanoscale. 2016;8:1868218692. doi:10.1039/c6nr07172d

3. Shen Z, Wu H, Yang S, et al. A novel Trojan-horse targeting strategy to reduce the non-specific uptake of nanocarriers by non-cancerous cells. Biomaterials. 2015;70:1-11. doi:10.1016/j.biomaterials.2015.08.022

4. Zaghloul H, Pallayova M, Al-Nuaimi O, Hovis KR, Taheri S. Association between diabetes mellitus and olfactory dysfunction: current perspectives and future directions. Diabetic Med. 2018;35:41-52. doi:10.1111/dme.13542

5. Forbes JM, Cooper ME. Mechanisms of diabetic complications. Physiol Rev. 2013;93:137-188. doi:10.1152/physrev.00045.2011

6. Zhou C, Teegala SB, Khan BA, Gonzalez C, Routh VH. Hypoglycemia: role of hypothalamic Glucose-Inhibited (GI) neurons in detection and correction. Front Physiol. 2018;9:192. doi:10.3389/ fphys.2018.00192

7. Siderowf A, Jennings D, Eberly S, et al. Impaired olfaction and other prodromal features in the Parkinson at-risk syndrome study. Mov Disord. 2012;27:406-412. doi:10.1002/mds.24892

8. Gray AJ, Staples V, Murren K, Dhariwal A, Bentham P. Olfactory identification is impaired in clinic-based patients with vascular dementia and senile dementia of Alzheimer type. Int $J$ Geriatr Psychiatry. 2001;16:513-517.

9. Lutterotti A, Vedovello M, Reindl M, et al. Olfactory threshold is impaired in early, active multiple sclerosis. Mult Scler. 2011;17:964969. doi: $10.1177 / 1352458511399798$

10. Gouveri E, Katotomichelakis M, Gouveris H, Danielides V, Maltezos E, Papanas N. Olfactory dysfunction in type 2 diabetes mellitus: an additional manifestation of microvascular disease? Angiology. 2014;65:869-876. doi:10.1177/0003319714520956

11. Brady S, Lalli P, Midha N, et al. Presence of neuropathic pain may explain poor performances on olfactory testing in diabetes mellitus patients. Chem Senses. 2013;38:497-507. doi:10.1093/chemse/bjt013

12. Wautier M-P, Massin P, Guillausseau P, et al. N(carboxymethyl)lysine as a biomarker for microvascular complications in type 2 diabetic patients. Diabetes Metab. 2003;29:44-52.

13. Sun Q, Tang DD, Yin EG, et al. Diagnostic significance of serum levels of nerve growth factor and brain derived neurotrophic factor in diabetic peripheral neuropathy. Med Sci Monit. 2018;24:5943-5950. doi:10.12659/MSM.909449
14. Hernandez C, Bogdanov P, Corraliza L, et al. Topical administration of GLP-1 receptor agonists prevents retinal neurodegeneration in experimental diabetes. Diabetes. 2016;65:172-187. doi:10.2337/db15-0443

15. Simo R, Hernandez C. GLP-1R as a target for the treatment of diabetic retinopathy: friend or foe? Diabetes. 2017;66:1453-1460. doi:10.2337/db16-1364

16. Hernandez C, Bogdanov P, Sola-Adell C, et al. Topical administration of DPP-IV inhibitors prevents retinal neurodegeneration in experimental diabetes. Diabetologia. 2017;60:2285-2298. doi:10.1007/s00125017-4388-y

17. Han HD, Jeon YW, Kwon HJ, et al. Therapeutic efficacy of doxorubicin delivery by a $\mathrm{CO} 2$ generating liposomal platform in breast carcinoma. Acta Biomater. 2015;24:279-285. doi:10.1016/j. actbio.2015.06.019

18. Naeini AT, Adeli M, Vossoughi M. Poly(citric acid)-block-poly(ethylene glycol) copolymers-new biocompatible hybrid materials for nanomedicine. Nanomedicine. 2010;6:556-562. doi:10.1016/j. nano.2009.11.008

19. Lee DP, Deonarine AS, Kienetz M, et al. A novel pathway for lipid biosynthesis: the direct acylation of glycerol. $J$ Lipid Res. 2001;42:1979-1986.

20. Adeli M, Rasoulian B, Saadatmehr F, Zabihi F. Hyperbranched poly(citric acid) and its application as anticancer drug delivery system. J Appl Polym Sci. 2013;129:3665-3671. doi:10.1002/ app. 39028

21. Vigo E, Cepeda A, Gualillo O, Perez-Fernandez R. In-vitro antiinflammatory effect of Eucalyptus globulus and Thymus vulgaris: nitric oxide inhibition in J774A.1 murine macrophages. $J$ Pharm Pharmacol. 2004;56:257-263. doi:10.1211/0022357022665

22. Martí D, Villagrasa V, Martinez-Solís I, Blanquer A, Castillo E, Royo LM. Hystological and pharmacological study of Thymus piperella (L.). Phytother Res. 2005;19:298-302. doi:10.1002/ptr.1569

23. Boskabady MH, Aslani MR, Kiani S. Relaxant effect of Thymus vulgaris on guinea-pig tracheal chains and its possible mechanism (s). Phytother Res. 2006;20:28-33. doi:10.1002/ptr.1796

24. Wattanasatcha A, Rengpipat S, Wanichwecharungruang S. Thymol nanospheres as an effective anti-bacterial agent. Int $J$ Pharm. 2012;434:360-365. doi:10.1016/j.ijpharm.2012.06.017

25. Dhaneshwar S, Patel V, Patil D, Meena G. Studies on synthesis, stability, release and pharmacodynamic profile of a novel diacereinthymol prodrug. Bioorg Med Chem Lett. 2013;23:55-61. doi:10.1016/j.bmcl.2012.11.016

26. Saravanan S, Pari L. Protective effect of thymol on high fat diet induced diabetic nephropathy in C57BL/6J mice. Chem Biol Interact. 2016;245:1-11. doi:10.1016/j.cbi.2015.11.033

27. Robledo N, Vera P, Lopez L, Yazdani-Pedram M, Tapia C, Abugoch L. Thymol nanoemulsions incorporated in quinoa protein/chitosan edible films; antifungal effect in cherry tomatoes. Food Chem. 2018;246:211-219. doi:10.1016/j.foodchem.2017.11.032

28. Jafari A, Rasmi Y, Hajaghazadeh M, Karimipour M. Hepatoprotective effect of thymol against subchronic toxicity of titanium dioxide nanoparticles: biochemical and histological evidences. Environ Toxicol Pharmacol. 2018;58:29-36. doi:10.1016/j. etap.2017.12.010

29. Dong Q, Gu L. Synthesis of AN-g-casein copolymer in concentrated aqueous solution of sodium thiocyanate and AN-g-casein fiber's structure and property. Eur Polym J. 2002;38:511-519. doi:10.1016/ S0014-3057(01)00214-2

30. Neri D, Szyperski T, Otting G, Senn H, Wuethrich K. Stereospecific nuclear magnetic resonance assignments of the methyl groups of valine and leucine in the DNA-binding domain of the 434 repressor by biosynthetically directed fractional carbon-13 labeling. Biochemistry. 1989;28:7510-7516. doi:10.1021/bi00445a003 
31. Lee WY. Calibration of the gel permeation chromatography polyester resins. $J$ Appl Polym Sci. 1978;22:3343-3344. doi:10.1002/ app.1978.070221128

32. Brown W, Schillen K, Hvidt S. Triblock copolymers in aqueous solution studied by static and dynamic light scattering and oscillatory shear measurements: influence of relative block sizes. $J$ Phys Chem. 1992;96:6038-6044. doi:10.1021/j100193a072

33. Rezayati Charani P, Dehghani-Firouzabadi M, Afra E, Shakeri A. Rheological characterization of high concentrated MFC gel from kenaf unbleached pulp. Cellulose. 2013;20:727-740. doi:10.1007/ s10570-013-9862-1

34. Lopez A, de Tangil MS, Vega-Orellana O, Ramirez AS, Rico M Phenolic constituents, antioxidant and preliminary antimycoplasmic activities of leaf skin and flowers of Aloe vera (L.) Burm. f. (syn. A barbadensis Mill.) from the Canary Islands (Spain). Molecules. 2013;18:4942-4954. doi:10.3390/molecules 18054942

35. Aghamohammadi A, Azadbakht M, Hosseinimehr SJ. Quantification of thymol content in different extracts of Zataria multiflora by HPLC method. Pharm Biomed Res. 2016;2:8-13. doi:10.18869/acadpub. pbr.2.1.8

36. Moayeri A, Azimi M, Karimi E, Aidy A, Abbasi N. Attenuation of morphine withdrawal syndrome by prosopis farcta extract and its bioactive component luteolin in comparison with clonidine in rats. Med Sci Monit Basic Res. 2018;24:151-158. doi:10.12659/ MSMBR.909930

37. Adams ML, Lavasanifar A, Kwon GS. Amphiphilic block copolymers for drug delivery. $J$ Pharm Sci. 2003;92:1343-1355. doi:10.1002/jps.10397

38. Lv Q, Yu A, Xi Y, et al. Development and evaluation of penciclovirloaded solid lipid nanoparticles for topical delivery. Int $J$ Pharm. 2009;372:191-198. doi:10.1016/j.ijpharm.2009.01.014

39. Majumdar D, Jung K-H, Zhang $\mathrm{H}$, et al. Luteolin nanoparticle in chemoprevention: in vitro and in vivo anticancer activity. Cancer Prev Res (Phila). 2014;7:65-73. doi:10.1158/1940-6207.CAPR-130230

40. Qiao M, Chen D, Ma X, Liu Y. Injectable biodegradable temperatureresponsive PLGA-PEG-PLGA copolymers: synthesis and effect of copolymer composition on the drug release from the copolymerbased hydrogels. Int J Pharm. 2005;294:103-112. doi:10.1016/j. ijpharm.2005.01.017

41. Azizi M, Bakhtiari M, Farahmand Ghavi F, Zandi M, Imani M, Joghataei MT. Purity determining of cultured OECs from olfactory mucosa of rats' pups. J Basic Res Med Sci. 2016;3:12-21. doi:10.18869/acadpub.jbrms.3.4.12

42. Yee SB, Lee JH, Chung HY, et al. Inhibitory effects of luteolin isolated from Ixeris sonchifolia Hance on the proliferation of HepG2 human hepatocellular carcinoma cells. Arch Pharm Res. $2003 ; 26: 151-156$.

43. Johnson JL, Gonzalez de Mejia E. Interactions between dietary flavonoids apigenin or luteolin and chemotherapeutic drugs to potentiate anti-proliferative effect on human pancreatic cancer cells, in vitro. Food Chem Toxicol. 2013;60:83-91. doi:10.1016/j. fct.2013.07.036

44. Green LC, Wagner DA, Glogowski J, Skipper PL, Wishnok JS, Tannenbaum SR. Analysis of nitrate, nitrite, and $[15 \mathrm{~N}]$ nitrate in biological fluids. Anal Biochem. 1982;126:131-138. doi:10.1016/ 0003-2697(82)90118-x

45. Cosentino F, Hishikawa K, Katusic ZS, Luscher TF. High glucose increases nitric oxide synthase expression and superoxide anion generation in human aortic endothelial cells. Circulation. 1997;96:25-28. doi:10.1161/01.cir.96.1.25

46. Mansouri N, Rikhtegar N, Panahi H, Atabi F, Karimi Shahraki B. Porosity, characterization and structural properties of natural zeolite Clinoptilolite - As a sorbent. environment protection engineering. 2013;39:139-152.
47. Alvarez Echazu MI, Olivetti CE, Anesini C, Perez CJ, Alvarez GS, Desimone MF. Development and evaluation of thymol-chitosan hydrogels with antimicrobial-antioxidant activity for oral local delivery. Mater Sci Eng C Mater Biol Appl. 2017;81:588-596. doi:10.1016/j.msec.2017.08.059

48. Byrne JD, Betancourt T, Brannon-Peppas L. Active targeting schemes for nanoparticle systems in cancer therapeutics. Adv Drug Deliv Rev. 2008;60:1615-1626. doi:10.1016/j.addr.2008.08.005

49. Kolhe P, Misra E, Kannan RM, Kannan S, Lieh-Lai M. Drug complexation, in vitro release and cellular entry of dendrimers and hyperbranched polymers. Int J Pharm. 2003;259:143-160.

50. Zhang H, Zhao C, Cao H, et al. Hyperbranched poly(amine-ester) based hydrogels for controlled multi-drug release in combination chemotherapy. Biomaterials. 2010;31:5445-5454. doi:10.1016/j. biomaterials.2010.03.034

51. Rokicki G, Rakoczy P, Parzuchowski P, Sobiecki M. Hyperbranched aliphatic polyethers obtained from environmentally benign monomer: glycerol carbonate. Green Chem. 2005;7:529-539.

52. Zhu Z, Kai L, Wang Y. Synthesis and applications of hyperbranched polyesters - preparation and characterization of crystalline silver nanoparticles. Mater Chem Phys. 2006;96:447-453. doi:10.1016/j. matchemphys.2005.07.067

53. Wang T, Li M, Gao H, Wu Y. Nanoparticle carriers based on copolymers of poly(epsilon-caprolactone) and hyperbranched polymers for drug delivery. J Colloid Interface Sci. 2011;353:107-115. doi:10.1016/j.jcis.2010.09.053

54. Seiler M. Hyperbranched polymers: phase behavior and new applications in the field of chemical engineering. fluid phase equilibria. 2006;241. doi:10.1148/radiol.2413051535

55. Saravanan S, Malathi S, Sesh PSL, Selvasubramarian S, Balasubramarian S, Pandiyan V.Hydrophilic poly (ethylene glycol) capped poly (lactic-co-glycolic) acid nanoparticles for subcutaneous delivery of insulin in diabetic rats. Int $J$ Biol Macromol. 2017;95:1190-1198. doi:10.1016/j.ijbiomac.2016.11.009

56. Dulak J, Deshane J, Jozkowicz A, Agarwal A. Heme oxygenase-1 and carbon monoxide in vascular pathobiology: focus on angiogenesis. Circulation. 2008;117:231-241. doi:10.1161/CIRCULATIONA HA.107.698316

57. Meissner G. Ryanodine activation and inhibition of the $\mathrm{Ca} 2+$ release channel of sarcoplasmic reticulum. J Biol Chem. 1986;261:6300-6306.

58. Verma A, Uzun $\mathrm{O}, \mathrm{Hu} \mathrm{Y}$, et al. Surface-structure-regulated cellmembrane penetration by monolayer-protected nanoparticles. Nat Mater. 2008;7:588-595. doi:10.1038/nmat2202

59. Medina E, Caro N, Abugoch L, Gamboa A, Díaz-Dosque M, Tapia C. Chitosan thymol nanoparticles improve the antimicrobial effect and the water vapour barrier of chitosan-quinoa protein films. J Food Eng. 2019;240:191-198. doi:10.1016/j.jfoodeng.2018.07.023

60. Yang R, Yang SG, Shim WS, et al. Lung-specific delivery of paclitaxel by chitosan-modified PLGA nanoparticles via transient formation of microaggregates. J Pharm Sci. 2009;98:970-984. doi:10.1002/jps.21487

61. Hariharan S, Bhardwaj V, Bala I, Sitterberg J, Bakowsky U, Kumar MNVR. Design of estradiol loaded PLGA nanoparticulate formulations: a potential oral delivery system for hormone therapy. Pharm Res. 2006;23:184-195.

62. Patel T, Zhou J, Piepmeier JM, Saltzman WM. Polymeric nanoparticles for drug delivery to the central nervous system. Adv Drug Deliv Rev. 2012;64:701-705. doi:10.1016/j.addr.2011.12.006

63. Li KK, Yin SW, Yang XQ, Tang CH, Wei ZH. Fabrication and characterization of novel antimicrobial films derived from thymolloaded zein-sodium caseinate (SC) nanoparticles. J Agric Food Chem. 2012;60:11592-11600. doi:10.1021/jf302752v

64. Sotelo-Boyas M, Correa-Pacheco Z, Bautista-Banos S, Gomez YGY. Release study and inhibitory activity of thyme essential oil-loaded chitosan nanoparticles and nanocapsules against foodborne bacteria. Int J Biol Macromol. 2017;103:409-414. doi:10.1016/j.ijbiomac.2017.05.063 
65. Pivetta TP, Simoes S, Araujo MM, Carvalho T, Arruda C, Marcato PD. Development of nanoparticles from natural lipids for topical delivery of thymol: investigation of its anti-inflammatory properties. Colloids Surf B Biointerfaces. 2018;164:281-290. doi:10.1016/j. colsurfb.2018.01.053

66. Mattos BD, Tardy BL, Pezhman M, et al. Controlled biocide release from hierarchically-structured biogenic silica: surface chemistry to tune release rate and responsiveness. Sci Rep. 2018;8:5555. doi:10.1038/s41598-018-23921-2

67. Duelli R, Maurer MH, Staudt R, Heiland S, Duembgen L, Kuschinsky W. Increased cerebral glucose utilization and decreased glucose transporter Glut1 during chronic hyperglycemia in rat brain. Brain Res. 2000;858:338-347. doi:10.1016/s0006-8993(00)01942-9

68. Malone JI, Hanna S, Saporta S, et al. Hyperglycemia not hypoglycemia alters neuronal dendrites and impairs spatial memory. Pediatr Diabetes. 2008;9:531-539. doi:10.1111/j.1399-5448.2008.00431.x

69. Summers WK. Alzheimer's disease, oxidative injury, and cytokines. $J$ Alzheimer Dis. 2004;6:651-657; discussion 673-681.

70. Pasquier F, Boulogne A, Leys D, Fontaine P. Diabetes mellitus and dementia. Diabetes Metab. 2006;32:403-414. doi:10.1016/S12623636(07)70298-7

71. Baron-Menguy C, Bocquet A, Guihot AL, et al. Effects of red wine polyphenols on postischemic neovascularization model in rats: low doses are proangiogenic, high doses anti-angiogenic. Faseb $J$. 2007;21:3511-3521. doi:10.1096/fj.06-7782com

72. Jukic M, Politeo O, Maksimovic M, Milos M, Milos M. In vitro acetylcholinesterase inhibitory properties of thymol, carvacrol and their derivatives thymoquinone and thymohydroquinone. Phytother Res. 2007;21:259-261. doi:10.1002/ptr.2063

73. Garcia DA, Bujons J, Vale C, Sunol C. Allosteric positive interaction of thymol with the GABAA receptor in primary cultures of mouse cortical neurons. Neuropharmacology. 2006;50:25-35. doi:10.1016/j. neuropharm.2005.07.009

74. Borah D, Deka P, Bhattacharjee P, Changmai A, Yadav RNS. Ocimum sanctum mediated silver nano particles showed better antimicrobial activities compared to citrate stabilized silver nano particles against multidrug resistant bacteria. J Pharm Res. 2013;7:478-482.

75. Russell JW, Golovoy D, Vincent AM, et al. High glucose-induced oxidative stress and mitochondrial dysfunction in neurons. Faseb $J$. 2002;16:1738-1748. doi:10.1096/fj.01-1027com

76. Huang Y, Leng TD, Inoue K, et al. TRPM7 channels play a role in high glucose-induced endoplasmic reticulum stress and neuronal cell apoptosis. J Biol Chem. 2018;293:14393-14406. doi:10.1074/jbc.RA117.001032

77. Ghafourifar P, Schenk U, Klein SD, Richter C. Mitochondrial nitric-oxide synthase stimulation causes cytochrome $\mathrm{c}$ release from isolated mitochondria. Evidence for intramitochondrial peroxynitrite formation. $J$ Biol Chem. 1999;274:31185-31188. doi:10.1074/jbc.274.44.31185

78. Brown GC. Regulation of mitochondrial respiration by nitric oxide inhibition of cytochrome $\mathrm{c}$ oxidase. Biochim Biophys Acta. 2001;1504:46-57. doi:10.1016/s0005-2728(00)00238-3

79. Wright G, Terada K, Yano M, Sergeev I, Mori M. Oxidative stress inhibits the mitochondrial import of preproteins and leads to their degradation. Exp Cell Res. 2001;263:107-117. doi:10.1006/ excr.2000.5096
80. Kowaltowski AJ, Castilho RF, Vercesi AE. Mitochondrial permeability transition and oxidative stress. FEBS Lett. 2001;495:12-15. doi:10.1016/s0014-5793(01)02316-x

81. Yamazaki M, Sakura N, Chiba K, Mohri T. Prevention of the neurotoxicity of the amyloid beta protein by genipin. Biol Pharm Bull. 2001;24:1454-1455. doi:10.1248/bpb.24.1454

82. Nam KN, Choi YS, Jung HJ, et al. Genipin inhibits the inflammatory response of rat brain microglial cells. Int Immunopharmacol. 2010;10:493-499. doi:10.1016/j.intimp.2010.01.011

83. Erukainure OL, Oyebode OA, Ibeji CU, Koorbanally NA, Islam MS. Vernonia Amygdalina Del. stimulated glucose uptake in brain tissues enhances antioxidative activities; and modulates functional chemistry and dysregulated metabolic pathways. Metab Brain Dis. 2019;34:721-732. doi:10.1007/s11011-018-0363-7

84. Rahman MM, Islam MB, Biswas M, Khurshid Alam AHM. In vitro antioxidant and free radical scavenging activity of different parts of Tabebuia pallida growing in Bangladesh. BMC Res Notes. 2015;8:621. doi:10.1186/s13104-015-1618-6

85. Friedrich EB, Liu E, Sinha S, et al. Integrin-linked kinase regulates endothelial cell survival and vascular development. Mol Cell Biol. 2004;24:8134-8144. doi:10.1128/MCB.24.18.8134-8144.2004

86. Li Y-J, Hui Y-N, Yan F, Du Z-J. Up-regulation of integrin-linked kinase in the streptozotocin-induced diabetic rat retina. Graefe's Arch Clin Exp Ophthalmol. 2007;245:1523-1532. doi:10.1007/s00417007-0616-3

87. Wautier MP, Massin P, Guillausseau PJ, et al. N(carboxymethyl) lysine as a biomarker for microvascular complications in type 2 diabetic patients. Diabetes Metab. 2003;29:44-52. doi:10.1016/ S1262-3636(07)70006-X

88. Nelson TJ, Sun MK, Hongpaisan J, Alkon DL. Insulin, PKC signaling pathways and synaptic remodeling during memory storage and neuronal repair. Eur J Pharmacol. 2008;585:76-87. doi:10.1016/j. ejphar.2008.01.051

89. Riaz A, Rasul A, Hussain G, et al. Astragalin: a bioactive phytochemical with potential therapeutic activities. Adv Pharmacol Sci. 2018;2018:9794625. doi:10.1155/2018/9794625

90. Saravanan S, Pari L. Role of thymol on hyperglycemia and hyperlipidemia in high fat diet-induced type 2 diabetic C57BL/6J mice. Eur J Pharmacol. 2015;761:279-287. doi:10.1016/j.ejphar.2015.05.034

91. Zhu H, Yu WJ, Le Y, et al. High glucose levels increase the expression of neurotrophic factors associated with p-p42/p44 MAPK in Schwann cells in vitro. Mol Med Rep. 2012;6:179-184. doi: $10.3892 / \mathrm{mmr} .2012 .896$

92. Dong Y, Feng Z-L, Chen H-B, Wang F-S, Lu J-H. Corni Fructus: a review of chemical constituents and pharmacological activities. Chin Med. 2018;13:34. doi:10.1186/s13020-018-0191-z

93. Ma P, Mao X-Y, Li X. L, et al. Baicalin alleviates diabetes associated cognitive deficits via modulation of mitogen-activated protein kinase signaling, brain derived neurotrophic factor and apoptosis. Mol Med Rep. 2015;12:-6377-6383. doi:10.3892/mmr.2015.4219

94. Comelli F, Bettoni I, Colleoni M, Giagnoni G, Costa B. Beneficial effects of a Cannabis sativa extract treatment on diabetes-induced neuropathy and oxidative stress. Phytother Res. 2009;23:1678-1684. doi:10.1002/ptr.2806
Drug Design, Development and Therapy

\section{Publish your work in this journal}

Drug Design, Development and Therapy is an international, peerreviewed open-access journal that spans the spectrum of drug design and development through to clinical applications. Clinical outcomes, patient safety, and programs for the development and effective, safe, and sustained use of medicines are a feature of the journal, which has also been accepted for indexing on PubMed Central. The manuscript management system is completely online and includes a very quick and fair peer-review system, which is all easy to use. Visit http://www. dovepress.com/testimonials.php to read real quotes from published authors. 